\title{
MULTIPLICATIONS ON COHOMOLOGY THEORIES WITH COEFFICIENTS
}

BY

\author{
ALVIN FRANK MARTIN
}

\begin{abstract}
Araki and Toda have considered the existence and classification of multiplications on generalized cohomology theories with coefficients in the category of finite CW-complexes. We consider the same matters for representable cohomology theories in a category of stable CW-spectra, such as that constructed by Adams. We obtain similar, and in certain instances stronger, results than Araki and Toda, with methods of proof that are often simpler and more straightforward.
\end{abstract}

Introduction. We suppose we are given a ring spectrum $E$ with multiplication (pairing) $\mu: E \wedge E \rightarrow E$ representing a multiplicative cohomology theory. Whenever necessary we assume that $\mu$ is commutative or associative. (Actually slightly weaker conditions suffice.) The corresponding theory with $Z_{q}$-coefficients is then represented by $E \wedge M_{q}$, where $M_{q}$ is the Moore spectrum for $Z_{q}$. We then consider multiplications $\mu_{q}:\left(E \wedge M_{q}\right) \wedge\left(E \wedge M_{q}\right)$ $\rightarrow E \wedge M_{q}$ on the theory with coefficients which, in an appropriate sense, via the reduction of coefficients morphism, are compatible with $\mu$. We consider also various additional properties which such $\mu_{q}$ may possess, including those of being Bockstein (i.e., making the Bockstein morphism an antiderivation), commutativity and associativity.

Our main existence results may be summarized as follows. If $q$ is odd there is always a canonical compatible multiplication $\mu_{q}$ which is Bockstein and commutative. This multiplication is also associative if $q$ is not a multiple of three, or if a certain condition on $E$ involving $\nu: S^{3} \rightarrow S^{0}$, the generator of the stable 3 -stem, is satisfied. If $q \equiv 0(\bmod 4)$ there are in general two such canonical, but not canonically distinguishable, compatible multiplications which are Bockstein. We determine a condition on $E$ involving $\eta: S^{1} \rightarrow S^{0}$, the generator of the stable 1-stem, which assures that these multiplications are commutative, and conditions involving $\eta$ and $\nu$ which assure that they are associative. If $q \equiv 2(\bmod 4)$ we determine a condition on $E$, involving $\eta$, which is necessary and sufficient for the existence of a compatible multiplica-

Received by the editors October 5, 1977.

AMS (MOS) subject classifications (1970). Primary 55B20, 55B45; Secondary 55H25.

Key words and phrases. Generalized cohomology theory, spectra, multiplicative cohomology theory, generalized cohomology with $Z_{q}$ coefficients, Moore spectrum, Bockstein spectral sequence. 
tion, a stronger condition which is necessary and sufficient for the existence of a Bockstein compatible multiplication, and a yet stronger condition which is necessary and sufficient for the existence of a commutative (and Bockstein) compatible multiplication. There is no canonical choice of compatible multiplication in this case, and for some $E$ compatible multiplications do not exist. A Bockstein compatible multiplication is associative in this case if $q$ is not a multiple of three, or if a condition on $E$ involving $\nu$ (the same as the one needed when $q$ is an odd multiple of three) is satisfied.

As for classification, our broadest result is that the group of stable morphisms [ $E \wedge S^{1} \wedge E \wedge S^{1}, E \wedge M_{q}$ ] acts transitively without fixed points on the set of all compatible multiplications, provided this set is nonempty. We prove several other results of a similar type which classify all compatible multiplications having specified properties.

Finally we consider multiplicative structures on Bockstein spectral sequences in this context. We show that for each prime $p$ there are successively induced multiplications on each stage of the $\bmod p$ Bockstein spectral sequence which are compatible with $\mu$, commutative, associative, and with respect to which the differentials are antiderivations. Here we assume conditions involving $\eta$ for the case $p=2$ and, for associativity, a condition involving $\nu$ for the case $p=3$.

For the sake of brevity, we shall omit proofs similar to those of Araki and Toda ([3], [4]), and of some results whose proofs are quite technical and detailed. Full proofs may be found in [8].

Maunder [9] has some of the same results as [3] and [4], but these are limited to existence results and to odd $q$.

\section{PART I: BASIC IDEAS}

1. Preliminaries. We assume throughout that we are working in Adams' stable category of $\mathrm{CW}$-spectra [2], or a similar such category. Thus the objects, roughly, are spectra whose component spaces are CW-complexes. We can speak of the stable cells of such an object. Morphisms are defined in an appropriate way, and represent stable homotopy classes.

An important example of such a spectrum is $S^{i}$, the stable $i$-sphere, for any integer $i$. In terms of component spaces $\left(S^{i}\right)_{n}=S^{i+n}$ for $n \geqslant-i$.

The most important technical construction is that of the smash product $X \wedge Y$ of two spectra, having the usual properties of a smash product. In particular, for any spectrum $X$ there are canonical equivalences $S^{0} \wedge X \approx X$ $\approx X \wedge S^{0}$, where $S^{0}$ is the sphere spectrum. We shall make free use of these equivalences without explicit mention. (For details on this and other aspects of the category, see [2].)

We shall take our suspensions on the right. Thus $S X=X \wedge S^{1}$, and $S^{i+1} X=S\left(S^{i} X\right)$. 
We let $[X, Y]_{i}$ denote the morphisms from $X$ to $Y$ of degree $i$. If $i$ is unspecified, it will be assumed to be zero. Thus $[X, Y]_{i}=\left[S^{i} X, Y\right]$. Also for any particular morphism, if the degree is unspecified, it will be assumed to be zero.

We may sometimes identify a morphism with its suspension or desuspension. For example, we shall let $\eta$ denote the generator of the stable 1-stem, and we may write $\eta: S^{1} \rightarrow S^{0}$, or $\eta: S^{3} \rightarrow S^{2}$, or $\eta: S^{7} \rightarrow S^{6}$, etc.

For any spectrum $X$ let $1=1_{X}: X \rightarrow X$ denote the identity morphism, and $q=q_{X}=q \cdot 1_{X}: X \rightarrow X$, for any integer $q$. For any $X$ and $Y$ let $T=T_{X, Y}$ : $X \wedge Y \rightarrow Y \wedge X$ denote the switching morphism.

Our category also contains a wedge product $X \vee Y$ of any two spectra $X$ and $Y$, having the usual properties of a wedge product. Given $X=\bigvee_{i=1}^{m} X_{i}$, let $n_{i}: X_{i} \rightarrow X$ denote the inclusion of the $i$ th factor and $p_{i}: X \rightarrow X_{i}$ the projection onto the $i$ th component, $i=1, \ldots, m$.

Given a CW-complex $X$, we may identify $X$ with its suspension spectrum, defined by $(X)_{i}=S^{i} X, i>0$.

2. Cohomology with coefficients. Let $h$ be a generalized cohomology theory satisfying the wedge axiom. We let $h^{i}(x)$ denote the $i$ th reduced cohomology group of $X$ with respect to $h$. We shall never consider nonreduced cohomology groups. By Brown's theorem, $h$ is represented by a spectrum $E$. Thus $h^{i}(x) \approx[X, E]_{-i}$.

If $E$ represents $h$, we define the cohomology theory corresponding to $h$ with coefficients $Z_{q}$ to be the theory which is represented by the spectrum $E \wedge M_{q}$. Here $M_{q}$ denotes the Moore spectrum of $Z_{q}$. Thus $h^{i}\left(X ; Z_{q}\right) \approx[X, E \wedge$ $\left.M_{q}\right]_{-i}$. $M_{q}$ may be stably represented as $S^{0} U_{q} e^{1}$. Thus in terms of component spaces we have $\left(M_{q}\right)_{1}=S^{1} U_{q} e^{2}$ which is a Moore space for $Z_{q}$.

We have a cofiber sequence

$$
\stackrel{q}{\rightarrow} S^{0} \stackrel{i_{q}}{\rightarrow} M_{q} \stackrel{\pi_{q}}{\rightarrow} S^{1} \stackrel{q}{\rightarrow}
$$

where $i_{q}$ is the inclusion morphism of $S^{0}$, and $\pi_{q}$ collapses $S^{0}$.

A different definition is often given for generalized cohomology with coefficients in the category of finite $\mathrm{CW}$-complexes. Here $h^{i}\left(X ; Z^{q}\right)=$ $h^{i+2}\left(X \wedge\left(M_{q}\right)_{1}\right)$. One would expect these two definitions to coincide.

Proposition 1. For a finite $C W$-complex $X$ there is a natural equivalence $\left[X, E \wedge M_{q}\right]_{-i} \approx\left[X \wedge\left(M_{q}\right)_{1}, E\right]_{-(i+2)}$.

Proof. Apply Spanier-Whitehead duality to (1) to get a cofiber sequence

$$
\stackrel{q}{\rightarrow} S^{-1} \stackrel{\tilde{\pi}_{q}}{\rightarrow} \tilde{M}_{q} \stackrel{\tilde{i}_{q}}{\rightarrow} S^{0} \stackrel{q}{\rightarrow} \text {. }
$$

Then it is clear that $\tilde{M}_{q}, \tilde{\pi}_{q}$ and $\tilde{i}_{q}$ are desuspensions, respectively, of $M_{q}, i_{q}$, 
and $\pi_{q}$. Then we have

$$
\begin{aligned}
{\left[X, E \wedge M_{q}\right]_{-1} } & \approx\left[X \wedge \tilde{M}_{q}, E\right]_{-i} \\
& \approx\left[X \wedge M_{q}, E\right]_{-(i+1)} \approx\left[X \wedge\left(M_{q}\right)_{1}, E\right]_{-(i+2)}
\end{aligned}
$$

3. Multiplications. If $h$ is a generalized cohomology theory, a multiplication on $h$ is a pairing $\mu: h^{i}(X) \otimes h^{j}(Y) \rightarrow h^{i+j}(X \wedge Y)$, which is natural, bilinear, compatible with suspension, and has a two-sided unit. Suppose $h$ is represented by a $C W$-spectrum $E$. By naturality a multiplication on $h$ corresponds to a pair of morphisms

$$
\mu: E \wedge E \rightarrow E \quad \text { L: } S^{0} \rightarrow E
$$

such that the following diagrams commute.

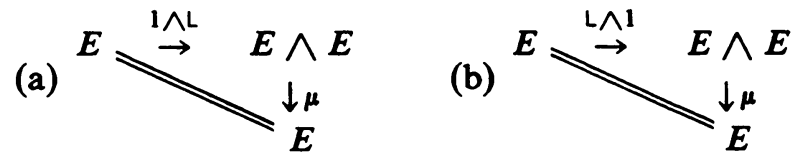

(Note that we use $\mu$ in two different, but related, ways.) We call $L$ the unit morphism of $\mu$. Often we omit mention of $L$.

$\mu$ is commutative if the following diagram commutes.

$$
\begin{array}{ccc}
E \wedge E & \stackrel{T}{\rightarrow} & E \wedge E \\
\mu \searrow & & \swarrow \mu
\end{array}
$$

$\mu$ is associative if the following diagram commutes.

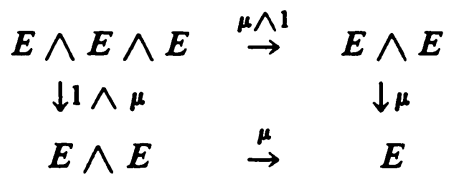

We say $\mu$ is commutative $(\bmod q)$ if the following diagram commutes.

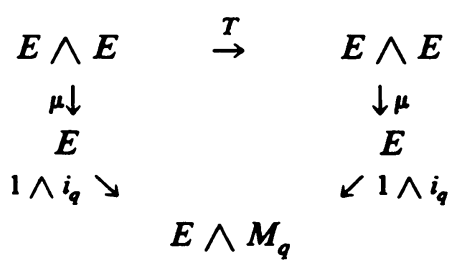

By (1) this is equivalent to requiring that $\mu-\mu T$ be in $q[E \wedge E, E]$. Associativity $(\bmod q)$ is defined analogously.

4. Compatible multiplications. We shall assume that we are given a multiplication $\mu$ on $E$, and shall then consider multiplications $\mu_{q}$ on $E \wedge M_{q}$. We shall ask of such a $\mu_{q}$, beside the usual properties, that it be compatible with $\mu$, 
meaning that the following diagrams commute:

(a)

$$
\begin{array}{cccc}
\text { (a) } \quad & E \wedge E \wedge M_{q} & \stackrel{1 \wedge i_{q} \wedge 1}{\rightarrow} & E \wedge M_{q} \wedge M_{q} \\
& \searrow \mu \wedge 1 & & \\
& E \wedge M_{q} \\
& E \wedge M_{q} \wedge E & \stackrel{1 \wedge i_{q}}{\rightarrow} & E \wedge M_{q} \wedge E \wedge M_{q} \\
\text { (b) } & \downarrow 1 \wedge T & & \downarrow \mu_{q} \\
& E \wedge E \wedge M_{q} \stackrel{\mu \wedge 1}{\rightarrow} & E \wedge M_{q}
\end{array}
$$

Proposition 2. If $\mu_{q}$ is such that (3) commutes, then $\mu_{q}$ is a multiplication with unit morphism $L \wedge i_{q}$.

Proof. From (3) we get commutative diagrams

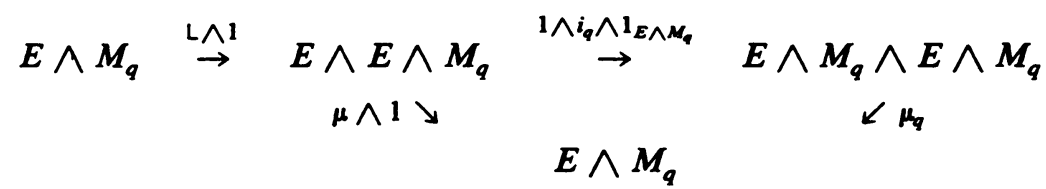

(b)

$$
\begin{array}{cccc}
E \wedge M_{q} \stackrel{1 \wedge L}{\rightarrow} & E \wedge M_{q} \wedge E & \stackrel{1 \wedge i_{q}}{\rightarrow} & E \wedge M_{q} \wedge E \wedge M_{q} \\
& \downarrow 1 \wedge T & & \downarrow \mu_{q} \\
& E \wedge E \wedge M_{q} \stackrel{\mu \wedge 1}{\rightarrow} & E \wedge M_{q}
\end{array}
$$

By (2)(a) and (4)(a), $L \wedge i_{q}$ is a left identity; by (2)(b) and (4)(b), it is a right identity.

(3) can be given another interpretation. Let us call $\rho_{q}=1 \wedge i_{q}: E \rightarrow E \wedge$ $M_{q}$ the reduction of coefficients morphism $\bmod q$. Given $\chi: X \rightarrow E$ of degree $-i$ representing $x \in h^{i}(X)$, we have a morphism $\rho_{q} X$ representing an element of $h^{i}\left(X ; Z_{q}\right)$. Denote this element $\rho_{q}(x)$. This defines the generalized reduction of coefficients homomorphism $\rho_{q}: h^{i}(X) \rightarrow h^{i}\left(X ; Z_{q}\right)$.

Now define morphisms

$$
\begin{aligned}
& \mu_{L}=\mu \wedge 1: E \wedge E \wedge M_{q} \rightarrow E \wedge M_{q} \text { and } \\
& \mu_{R}=(\mu \wedge 1)(1 \wedge T): E \wedge M_{q} \wedge E \rightarrow E \wedge M_{q} .
\end{aligned}
$$

In an obvious way, these morphisms determine pairings

$$
\mu_{L}: h^{i}(X) \otimes h^{j}\left(Y ; Z_{q}\right) \rightarrow h^{i+j}\left(X \wedge Y ; Z_{q}\right)
$$

and

$$
\mu_{R}: h^{i}\left(X ; Z_{q}\right) \otimes h^{j}(Y) \rightarrow h^{i+j}\left(X \wedge Y ; Z_{q}\right),
$$

called left multiplication and right multiplication, respectively. Then (3) says that $\mu_{L}=\mu_{q}\left(\rho_{q} \otimes 1\right)$ and $\mu_{R}=\left(1 \otimes \rho_{q}\right)($ see $[3$, p. 84] $)$. 
Given $\mu$, we shall find a necessary and sufficient condition for the existence of a compatible $\mu_{q}: E \wedge M_{q} \wedge E \wedge M_{q} \rightarrow E \wedge M_{q}$, and we shall classify all such $\mu_{q}$. Further, we shall discuss the existence and classification of compatible $\mu_{q}$ which satisfy various additional properties. Whenever necessary, we shall assume $\mu$ to be commutative $(\bmod q)$ or associative $(\bmod q)$.

We shall omit subscript $q$ 's where no confusion will result. Thus we may write $M$ for $M_{q}, i$ for $i_{q}$, etc., except where the context requires that $q$ be specified.

5. Additional properties. In ordinary cohomology the Bockstein operator $\beta_{q}$ : $H^{i}\left(X ; Z_{q}\right) \rightarrow H^{i+1}\left(X ; Z_{q}\right)$ is antiderivation. This means that

$$
(x y) \beta_{q}=\left(y \beta_{q}\right)+(-1)^{\operatorname{deg} Y}\left(x \beta_{q}\right) y \text {. }
$$

(It suits our conventions to write $\beta_{q}$ on the right.) We shall extend this notion to generalized theories.

Let $\delta=\delta_{q}=(i \wedge 1) \pi: M \rightarrow S M$. Then define the Bockstein morphism

$$
\beta=\beta_{q}=1 \wedge \delta: E \wedge M \rightarrow E \wedge S M \text {. }
$$

Given $\chi: X \rightarrow E \wedge M$ of degree $i$ representing $x \in h^{i}\left(X ; Z_{q}\right)$, let $\beta \chi$ represent $(x) \beta_{q} \in h^{i+1}\left(X ; Z_{q}\right)$. This defines the generalized Bockstein operator.

We say that a compatible multiplication $\mu_{q}$ is Bockstein if $\beta_{q}$ is an antiderivation with respect to $\mu_{q}$, i.e., if (5) holds where the multiplication is with respect to $\mu_{q}$.

LEMMA 3. Let $\mu_{q}: E \wedge M \wedge E \wedge M \rightarrow E \wedge M$ be a compatible multiplication. Then $\mu_{q}$ is Bockstein if and only if $\beta_{q} \mu_{q}: E \wedge M \wedge E \wedge M \rightarrow E \wedge S M$ is the sum of the morphisms

$$
\gamma_{1}=\left(\mu_{R} \wedge 1\right)\left(1_{E \wedge M \wedge 1} \wedge \pi\right) \text { and } \gamma_{2}=\gamma_{1} \bar{T}
$$

where $\bar{T}: E \wedge M \wedge E \wedge M \rightarrow E \wedge M \wedge E \wedge M$ interchanges the factors of $M$, and fixes the $E$ factors.

PROof. By general category theory arguments, $\mu_{q}$ is Bockstein if and only if $\beta_{q} \mu_{q}$ is the sum of

$$
\begin{aligned}
& \tilde{\gamma}_{1}=\left(\mu_{q} \wedge 1\right)\left(1_{E \wedge M} \wedge \beta_{q}\right) \text { and } \\
& \tilde{\gamma}_{2}=\left(\mu_{q} \wedge 1\right)\left(1_{E \wedge M} \wedge T_{S^{1}, E \wedge M}\right)\left(\beta_{q} \wedge 1_{E \wedge M}\right) .
\end{aligned}
$$

Using (3) it may be seen that $\gamma_{1}=\tilde{\gamma}_{1}$ and $\gamma_{2}=\tilde{\gamma}_{2}$.

Next we consider a weak form of associativity that a compatible multiplication may satisfy. We say that $\mu_{q}$ is quasi-associative $[3, \mathrm{p} .85]$ if given $x \in h^{i}\left(X ; Z_{q}\right), y \in h^{j}\left(Y ; Z_{q}\right)$ and $z \in h^{k}\left(Z ; Z_{q}\right)$ such that at least one of $x, y$ and $z$ is in the image of $\rho$, then $(x y) z=x(y z)$, where products are with respect to $\mu_{q}$. Quasi-associativity will turn out to be a useful concept in classifying multiplications. Also, given that $\mu$ is associative $(\bmod q)$ and 
commutative $(\bmod q)$, it will turn out to be relatively easy to find a quasiassociative, compatible $\mu_{q}$, while finding an associative $\mu_{q}$ is more difficult.

Translated into the language of spectra, quasi-associativity requires that three diagrams commute, one of which is

$$
\begin{array}{ccc}
E \wedge E \wedge M \wedge E \wedge M & \stackrel{1 \wedge \mu_{q}}{\rightarrow} & E \wedge E \wedge M \\
\downarrow \mu_{L} \wedge 1_{E \wedge M} & & \downarrow \mu_{L} \\
E \wedge M \wedge E \wedge M & \stackrel{\mu_{q}}{\rightarrow} & E \wedge M
\end{array}
$$

The other diagrams are similar.

LEMMA 4. If a compatible $\mu_{q}$ is quasi-associative, then $\mu$ is associative $(\bmod q)$.

Proof. By (6),

$$
\begin{aligned}
\mu_{q}\left(\mu_{L} \wedge 1_{E \wedge M}\right)(1 & \wedge 1 \wedge i \wedge 1 \wedge i) \\
& =\mu_{L}\left(1 \wedge \mu_{q}\right)(1 \wedge 1 \wedge i \wedge 1 \wedge i): E \wedge E \wedge E \rightarrow E \wedge M .
\end{aligned}
$$

But by (3) this is equivalent to

$$
(1 \wedge i) \mu(\mu \wedge 1)=(1 \wedge i) \mu(1 \wedge \mu): E \wedge E \wedge E \rightarrow E \wedge M
$$

as desired.

\section{PART II: EXISTENCE OF MUlTIPLICATIONS}

1. Some morphisms involving $M$. The following lemma will be very important for us.

LEMMA 5. If $q \neq 2(\bmod 4), 1_{M}$ is of order $q$. If $q \equiv 2(\bmod 4), 1_{M}$ is of order $2 q$ and $q_{M}=i \eta \pi: M \rightarrow M$, where $\eta: S^{1} \rightarrow S^{0}$ generates the stable one-stem.

Proof. See [3, p. 75].

COROLlaRY 6. For any $X$ and $i, h^{i}\left(X ; Z_{q}\right)$ is a $Z_{q}$ module if $q \neq 2(\bmod 4)$ and $a Z_{2 q}$ module if $q \equiv 2(\bmod 4)$.

Now let $a, q$ and $r$ be integers with $q, r \geqslant 2$, such that $a q \equiv 0(\bmod r)$. Let $a^{\prime}=a q / r$. Then it is easily checked that there is a unique morphism $\bar{a}$ : $M_{r} \rightarrow M_{q}$ such that the following diagrams commute.

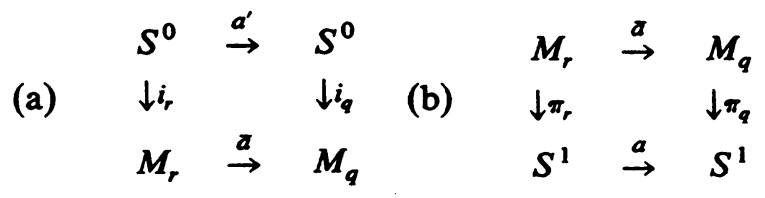


In particular we have morphisms $\bar{r}=\bar{r}_{q, r}: M_{q r} \rightarrow M_{q}$ and $\overline{1}=\overline{1}_{q, r}: M_{q} \rightarrow$ $M_{q r}$. It may be noted that these morphisms are Spanier-Whitehead dual to each other. Furthermore, using (7), the following relations are easily verified.

$$
\begin{aligned}
& \begin{array}{ll}
\text { (a) } i_{q}=\bar{r}_{q r}: S^{0} \rightarrow M_{q}, & \text { (c) } r i_{q r}=\overline{1}_{q}: S^{0} \rightarrow M_{q r}, \\
\text { (b) } \pi_{q}=\pi_{q r} \overline{1}: M_{q} \rightarrow S, & \text { (d) } m_{q r}=\pi_{q} \bar{r}: M_{q r} \rightarrow S ; \\
\text { (a) } \bar{r}_{q, r} \bar{s}_{q r, s}=\bar{r}_{q, r s}: M_{q r s} \rightarrow M_{q}, \\
\text { (b) } \overline{1}_{q r, s} \overline{1}_{q, r}=\overline{1}_{q, r s}: M_{q} \rightarrow M_{q r s} .
\end{array}
\end{aligned}
$$

Proposirion 7. For any spectra $A, B$ the short exact sequences based on (1)

(a) $0 \rightarrow[A, B] \otimes Z_{q}^{(1 \wedge i)} \stackrel{\rightarrow}{\rightarrow}[A, B \wedge M] \stackrel{(1 \wedge \pi)}{\rightarrow} \operatorname{Tor}\left(\left[A, B \wedge S^{\prime}\right], Z_{q}\right)$,

(b) $0 \rightarrow\left[A \wedge S^{\prime}, B\right] \otimes Z_{q} \stackrel{(1 \wedge \pi)^{*}}{\rightarrow}[A \wedge M, B]^{(1 \wedge i)^{*}} \rightarrow \operatorname{Tor}\left([A, B], Z_{q}\right) \rightarrow 0$ split if $q \equiv 2(\bmod 4)$ or if $q \equiv 2(\bmod 4)$ and $q_{B \wedge M}=0$ or $q_{A \wedge M}=0$. The proof is similar to [2, pp. 81-82].

We need to know the low dimensional homotopy and cohomotopy groups of $M_{q}$, and the morphisms of low degree from $M_{q}$ to itself. The following results come essentially from [3, pp. 95-97], but beware that in [3] $M_{q}$ means $\left(M_{q}\right)_{1}$, and thus the degrees are different.

The information in the following table is well known.

\begin{tabular}{|l||c|c|c|c|c|}
\multicolumn{1}{c||}{} & $i<0$ & $i=0$ & $i=1$ & $i=2$ & $i=3$ \\
\hline \hline$\left[S^{i}, S^{0}\right]$ & 0 & $Z$ & $Z_{2}$ & $Z_{2}$ & $Z_{24}$ \\
\hline generators & & 1 & $\eta$ & $\eta^{2}$ & $\nu$ \\
\hline
\end{tabular}

From (1) we get short exact sequences:

(a) $0 \rightarrow\left[S^{i}, S^{0}\right] \otimes Z_{q} \stackrel{i}{\rightarrow}\left[S^{i}, M_{q}\right] \stackrel{\pi}{\rightarrow} \operatorname{Tor}\left(\left[S^{i}, S^{1}\right], Z_{q}\right) \rightarrow 0$,

(b) $0 \rightarrow\left[S^{1}, S^{-i+1}\right] \otimes Z_{q} \stackrel{\pi^{*}}{\rightarrow}\left[M_{q}, S^{-i+1}\right] \stackrel{i *}{\rightarrow} \operatorname{Tor}\left(\left[S^{0}, S^{-i+1}\right], Z_{q}\right) \rightarrow 0$.

Proposition 8. The groups $\left[S^{i}, M_{q}\right]$ and $\left[M_{q}, S^{-i+1}\right]$ are given by the following table, $i<3$, where, for even $q, \tilde{\eta}=\tilde{\eta}_{q}: S^{2} \rightarrow M$ and $\bar{\eta}=\bar{\eta}_{q}: M \rightarrow$ $S^{-1}$ satisfy (a) $\pi \tilde{\eta}=\eta=\bar{\eta} i$, (b) $\overline{1}_{q, 2} k \tilde{\eta}_{q}=\tilde{\eta}_{2} k_{q}$, and (c) $\bar{n}_{q} \bar{k}_{q, 2} k=\bar{\eta}_{2} k_{q}$, for all positive integers $k$ and even positive integers $q$. 


\begin{tabular}{|l||c|c|c|c|c|}
\hline$\left[S^{i}, M_{q}\right] \approx\left[M_{q}, S^{-i+1}\right]$ & $i<-1$ & $i=0$ & $i=1$ & $i=2$ & $i=3$ \\
\hline \hline$q$ odd & 0 & $Z_{q}$ & 0 & 0 & $Z_{(q, 24)}$ \\
\hline$q \equiv 0(\bmod 4)$ & 0 & $Z_{q}$ & $Z_{2}$ & $Z_{2} \oplus Z_{2}$ & $Z_{q} \oplus Z_{(q, 24)}$ \\
\hline$q \equiv 2(\bmod 4)$ & 0 & $Z_{q} m$ & $Z_{2}$ & $Z_{4}$ & $Z_{2} \oplus Z_{(q, 24)}$ \\
\hline generators of $\left[S^{i}, M_{q}\right]$ & & $i$ & $i \eta$ & $\tilde{\eta}, i \eta^{2}$ & $\tilde{\eta} \eta, i v$ \\
\hline generators of $\left[M_{q}, S^{-i+1}\right]$ & & $\pi$ & $\eta \pi$ & $\bar{\eta}, \eta^{2} \pi$ & $\eta \bar{\eta}, v \pi$ \\
\hline
\end{tabular}

From (1) we get Spanier-Whitehead dual short exact sequences:
(a) $0 \rightarrow\left[S^{i} M_{q}, S^{0}\right] \otimes Z_{q} \stackrel{i_{*}}{\rightarrow}\left[S^{i} M_{q}, M_{q}\right] \stackrel{\pi_{*}}{\rightarrow} \operatorname{Tor}\left(\left[S^{i} M_{q}, S^{1}\right], Z_{q}\right) \rightarrow 0$,
(b) $0 \rightarrow\left[S^{i+1}, M_{q}\right] \otimes Z_{q} \stackrel{\pi^{*}}{\rightarrow}\left[S^{i} M_{q}, M_{q}\right] \stackrel{i^{*}}{\rightarrow} \operatorname{Tor}\left(\left[S^{i}, M_{q}\right], Z_{q}\right) \rightarrow 0$.

Proposition 9. The groups $\left[S^{i} M_{q}, M_{q}\right]$ are given by the following table for $i<1$.

\begin{tabular}{|c|c|c|c|c|}
\hline$\left[S^{i} M_{q}, M_{q}\right]$ & $i<-1$ & $i=-1$ & $i=0$ & $i=1$ \\
\hline$q$ odd & 0 & $Z_{q}$ & $Z_{q}$ & 0 \\
\hline generators & & $\delta$ & 1 & \\
\hline$q \equiv 0(\bmod 4)$ & 0 & $Z_{q}$ & $Z_{q} \oplus Z_{2}$ & $Z_{2} \oplus Z_{2} \oplus Z_{2}$ \\
\hline generators & & $\delta$ & $1, i \eta \pi$ & $\tilde{\eta} \pi, i \bar{\eta}, i \eta^{2} \pi$ \\
\hline$q \equiv 2(\bmod 4)$ & 0 & $Z_{q}$ & $Z_{2 q}$ & $Z_{2} \oplus Z_{2}$ \\
\hline generators & & $\delta$ & 1 & $\tilde{\eta} \pi, i \bar{\eta}$ \\
\hline
\end{tabular}

\section{A necessary condition.}

Proposition 10. A necessary condition for the existence of a compatible multiplication $\mu_{q}: E \wedge M \wedge E \wedge M \rightarrow E \wedge M$ is that $q_{E \wedge M}$ be trivial.

Proof. From (1) we get the exact sequence

$[E \wedge M \wedge E \wedge M, E \wedge M] \stackrel{\left(1_{E \wedge M \wedge E \wedge i)^{*}}^{\rightarrow}\right.}{\rightarrow}[E \wedge M \wedge E, E \wedge M] \stackrel{q}{\rightarrow}$.

If $\mu_{q} \in[E \wedge M \wedge E \wedge M, E \wedge M]$ is a compatible multiplication, then by (2) and (3),

$$
1_{E \wedge M}=\left(1_{E \wedge M} \wedge L\right)^{*}\left(1_{E \wedge M \wedge E} \wedge i\right)^{*} \mu_{q}
$$


Then from (12) it follows that $q \cdot 1_{E \wedge M}=q_{E \wedge M}=0$.

Notice that this is a condition on $E$, not on $\mu$. Thus given two different multiplications $\mu$ and $\mu^{\prime}$ on $E$, the impact of Proposition 10 on the possible existence of a compatible $\mu_{q}$ or $\mu_{q}^{\prime}$, respectively, is the same. This will be the case for all of the conditions we shall derive.

COROLlaRY 11. If there exists a compatible multiplication $\mu_{q}$, then for all $X$ and $i, h^{i}\left(X ; Z_{q}\right)$ is a $Z_{q}$ module.

For any morphism $\gamma: X \rightarrow Y$. Let $(\gamma)_{E}$ denote the morphism $1 \wedge \gamma$ : $E \wedge X \rightarrow E \wedge Y$. Let $[\gamma]_{E}$ denote the morphism $(L \wedge 1) \gamma: X \rightarrow E \wedge Y$.

LEMMA 12. $(\gamma)_{E}=0$ if and only if $[\gamma]_{E}=0$.

ProOF. This follows from the commutativity of the following diagrams.
$\begin{array}{ccc}X & \stackrel{\wedge}{\rightarrow} \quad E \wedge X \\ \text { (a) }[\gamma]_{E} \searrow & \downarrow(\gamma)_{E}\end{array}$
(b)

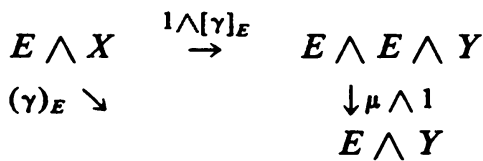

Proposition 13. If $q \equiv 2(\bmod 4)$ the following conditions are equivalent, and each is necessary for the existence of a compatible multiplication on $E \wedge M$.
(a) $(i \eta \pi)_{E}=0$,
(c) $(\eta \pi)_{E}=0$,
(e) $[i \eta]_{E}=0$,
(b) $(i \eta)_{E}=0$,
(d) $[i \eta \pi]_{E}=0$,
(f) $[\eta \pi]_{E}=0$.

Proof. That (a) is a necessary condition follows from Proposition 10 and Lemma 5. In view of Lemma 12, it suffices to show only that (a), (b) and (c) are equivalent. It is obvious that (b) and (c) each imply (a). Now suppose (a) holds. From (1) we get an exact sequence

$$
\stackrel{q}{\rightarrow}\left[E \wedge S^{1}, E \wedge M\right] \stackrel{(1 \wedge \pi)^{*}}{\rightarrow}[E \wedge M, E \wedge M]
$$

Since $1 \wedge i \eta$ is in the kernel of $(1 \wedge \pi)^{*}$, it must be a multiple of $q$, and thus of the form $q_{E \wedge M} \delta$ for some $\delta \in\left[E \wedge S^{1}, E \wedge M\right]$. But $q_{E \wedge M}=(i \eta \pi)_{E}=0$, so we conclude $1 \wedge i \eta=(i \eta)_{E}=0$. The proof that (b) implies (c) is similar.

Araki and Toda [3, p. 87] obtain a similar, but slightly different condition. Their condition is that $(\eta \pi)^{* *}=0$, which means that for any finite $\mathrm{CW}$-complex $X$ and any $\chi: X \rightarrow E$ (of arbitrary degree), $\chi(1 \wedge \eta \pi): X \wedge M \rightarrow E$ is trivial. Using Spanier-Whitehead duality in a manner similar to that in the proof of Proposition 1, we see this is equivalent to requiring that $(1 \wedge i \eta) x^{\prime}=$ 0 for any $\chi^{\prime}: X \rightarrow E \wedge S^{1}$ (of arbitrary degree). Our condition $(i \eta)_{E}=0$ is stronger because it does not require that $X$ be finite. This reflects the fact that our multiplications are defined on a larger category.

A similar distinction relative to [3] and [4] will exist with regard to other conditions that we shall derive. 
3. Multiplication generators. An obvious and important special case of the general problem is that of $E=S^{0}$, with $\mu=L=1 s^{0}$. Here we seek a multiplication $\tilde{\mu}_{q}: M \wedge M \rightarrow M$ with unit morphism $i$. Given such a $\tilde{\mu}_{q}$, we may define $\mu_{q}: E \wedge M \wedge E \wedge M \rightarrow E \wedge M$ by

$$
\mu_{q}=\left(\mu \wedge \tilde{\mu}_{q}\right)(1 \wedge T \wedge 1)
$$

LEMMA 14. If $\tilde{\mu}_{q}$ is such a compatible multiplication on $M$ and $\mu$ is associative $(\bmod q), \mu_{q}$ as defined in (13) is a compatible multiplication on $E \wedge M$, which is quasi-associative. If $\tilde{\mu}_{q}$ is Bockstein, then so is $\mu_{q}$. If $\tilde{\mu}$ is commutative and $\mu$ is commutative $(\bmod q)$, then $\mu_{q}$ is commutative. If $\tilde{\mu}_{q}$ is associative and $\mu$ is associative $(\bmod q)$, then $\mu_{q}$ is associative.

Proof. Check that all the appropriate diagrams commute.

LEMMA 15. If $q \equiv 2(\bmod 4)$, then there does not exist a compatible multiplication on $M$.

Proof. This is a consequence of Proposition 10 and Lemma 5.

We shall generalize the notion of defining $\mu_{q}$ from a multiplication $\tilde{\mu}_{q}$ on $M$. We shall say that a multiplication $\mu_{q}$ on $E \wedge M$ is proper if it is compatible and has the form

$$
\begin{aligned}
\mu_{q} & =\left(\mu_{L}\right)(\mu \wedge \tau)(1 \wedge T \wedge 1): E \wedge M \wedge E \wedge M \\
& \rightarrow E \wedge E \wedge M \wedge M \rightarrow E \wedge E \wedge M \rightarrow E \wedge M
\end{aligned}
$$

for some $\tau=\tau_{q}: M \wedge M \rightarrow E \wedge M$. We call such a $\tau$ a multiplication generator, and call $\mu_{q}=\mu(\tau)$ the multiplication generated by $\tau$.

If $\tilde{\mu}_{q}$ is a multiplication on $M_{q}$, and $\mu_{q}$ is defined from $\tilde{\mu}_{q}$ by (13), then $\mu_{q}$ is proper with multiplication generator $\tau=(L \wedge 1) \tilde{\mu}_{q}: M \wedge M \rightarrow E \wedge M$.

We say that a multiplication generator $\tau$ is Bockstein if $\beta \tau: M \wedge M \rightarrow E \wedge$ $S M$ is the sum of the morphisms $\tilde{\gamma}_{1}=L \wedge 1 \wedge \pi$ and $\tilde{\gamma}_{2}=\tilde{\gamma}_{1} T$. (Compare with Lemma 3.)

We say that a multiplication generator $\tau$ is commutative if the following diagram commutes.

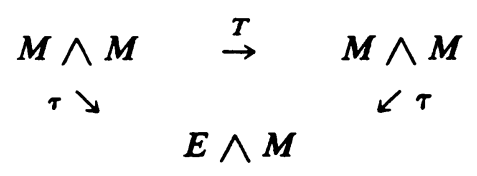

We say that a multiplication generator $\tau$ is associative if the following diagram commutes. 


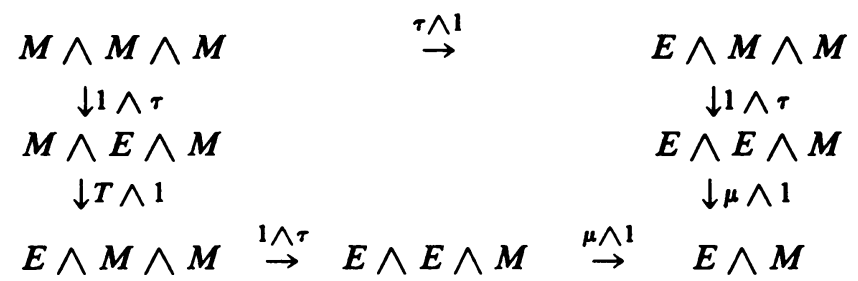

Let

(a) $\tau_{L}=(\mu \wedge 1)(1 \wedge \tau)(\tau \wedge 1): M \wedge M \wedge M \rightarrow E \wedge M$,

(b) $\tau_{R}=(\mu \wedge 1)(1 \wedge \tau)(T \wedge 1)(1 \wedge \tau): M \wedge M \wedge M \rightarrow E \wedge M$.

Proposition 16. (a) $A$ morphism $\tau: M \wedge M \rightarrow E \wedge M$ is a multiplication generator if and only if the following diagrams commute.

(a)

$$
\begin{array}{cc}
M \quad \stackrel{1 \wedge i}{\rightarrow} & M \wedge M \\
\searrow\llcorner\wedge 1 & \swarrow \tau \\
E \wedge M & \\
M \quad \stackrel{i \wedge 1}{\rightarrow} & M \wedge M \\
\searrow\llcorner\wedge 1 & \swarrow \tau \\
E \wedge M &
\end{array}
$$

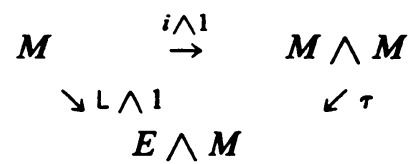

(b)

(b) If $\tau$ is a multiplication generator and $\mu$ is commutative $(\bmod q)$ and associative $(\bmod q)$, then $\mu(\tau)$ is quasi-associative. If in addition $\tau$ is associative, then $\mu(\tau)$ is associative.

(c) If $\mu$ is commutative $(\bmod q)$ and $\tau$ is commutative, then $\mu(\tau)$ is commutative.

(d) If $\mu$ is Bockstein, then $\mu(\tau)$ is Bockstein.

Proof. See [8, pp. 29-30].

Given a compatible multiplication $\mu_{q}: E \wedge M \wedge E \wedge M \rightarrow E \wedge M$, we can define its associated multiplication generator $\tau\left(\mu_{q}\right)$ by

$$
\tau\left(\mu_{q}\right)=\mu_{q}(L \wedge 1 \wedge\llcorner\wedge 1): M \wedge M \rightarrow E \wedge M \text {. }
$$

Proposition 17. (a) $\tau\left(\mu_{q}\right)$ is always a multiplication generator which is Bockstein if $\mu_{q}$ is Bockstein, commutative if $\mu_{q}$ is commutative, and associative if $\mu_{q}$ is associative.

(b) $\tau(\mu(\tau))=\tau$.

Proof. See [8, p. 31].

COROLLARY 18. If there exists a compatible multiplication $\mu_{q}$ on $E \wedge M$, then there is a proper multiplicatication $\mu_{q}^{\prime}$ on $E \wedge M$. If $\mu_{q}$ is Bockstein or is commutative, then $\mu_{q}^{\prime}$ is Bockstein or commutative, respectively. If $\mu$ is commutative $(\bmod q)$ and $\mu_{q}$ is associative then $\mu_{q}^{\prime}$ is associative. 
Proof. By Lemma 4 if $\mu_{q}$ is an associative compatible multiplication, then $\mu$ is associative $(\bmod q)$. The same may easily be shown for commutativity. Given $\mu_{q}$ we get a proper multiplication $\mu_{q}^{\prime}=\mu\left(\tau\left(\mu_{q}\right)\right)$ on $E \wedge M$. That $\mu_{q}^{\prime}$ has the same property as $\mu_{q}$ then follows in each case from Propositions 17 and 16.

4. The stable complex $M \wedge M$. It is important to have some understanding of the spectrum $M \wedge M$, and the switching morphism $T: M \wedge M \rightarrow M \wedge M$. The material in this section is similar to [3, pp. 98-108].

We define the spectrum $N=N_{q}$ as follows.

$$
N_{q}= \begin{cases}M_{q} \vee S M_{q} & \text { if } q \equiv 2(\bmod 4), \\ \left(M_{q} \vee S^{1}\right) U_{\gamma} e^{2} & \text { if } q \equiv 2(\bmod 4), \text { where } \gamma=n_{1} i \eta+n_{2} q .\end{cases}
$$

Next we define some morphisms involving $N$. Let $j_{0}: M \rightarrow N$ denote the inclusion of the subspectrum of $M$ (in either case), and let $k_{0}: N \rightarrow S M$ denote the morphism collapsing the subspectrum $M$. Let $j_{1}: S^{1} \rightarrow N$ denote $n_{2}\left(\llcorner\wedge 1): S^{1} \rightarrow S M \rightarrow N\right.$ if $q \neq 2(\bmod 4)$, and let it denote the inclusion of the subspectrum $S^{1}$ if $q \equiv 2(\bmod 4)$. Let $k_{1}: N \rightarrow S^{1}$ denote $\pi p_{1}: N \rightarrow M \rightarrow$ $S^{1}$ if $q \neq 2(\bmod 4)$, and if $q \equiv 2(\bmod 4)$ let it denote the morphism collapsing the subspectrum $\left(S^{0} \vee S^{1}\right) U_{\gamma^{\prime}} e^{2}$, where $S^{0}$ is regarded as included in $M$ by $i: S^{0} \rightarrow M$, and $\gamma^{\prime}=n_{1} \eta+n_{2} q: S^{1} \rightarrow S^{0} \vee S^{1}$. This is well defined since $\pi: M \rightarrow S^{1}$ may be regarded as the morphism collapsing the subspectrum $S^{0}$ of $M$.

Note that we have

$$
\begin{array}{ll}
\text { (a) } k_{1} j_{0}=\pi: M \rightarrow S^{1}, & \text { (b) } k_{0} j_{1}=i: S^{1} \rightarrow S M
\end{array}
$$

Proposition 19. (a) There exists an equivalence $\alpha: M \wedge M \rightarrow N$ such that the following diagram commutes.

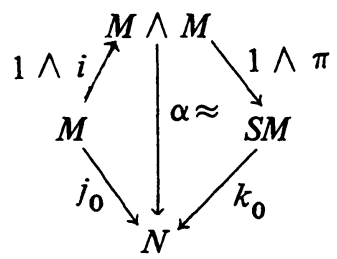

(b) A morphism $\alpha^{\prime}: M \wedge M \rightarrow N$ satisfies the same conditions as $\alpha$ if and only if $\alpha^{\prime}=\left(1+j_{0} \gamma k_{0}\right) \alpha$ for some $\gamma: S M \rightarrow M$.

(c) If $q$ is odd, then $\alpha$ is unique.

Now suppose $\alpha: M \wedge M \rightarrow N$ satisfies the conditions of Proposition 19 (a). We define a morphism $T_{\alpha}: N \rightarrow N$ by the commutativity of the following diagram. 


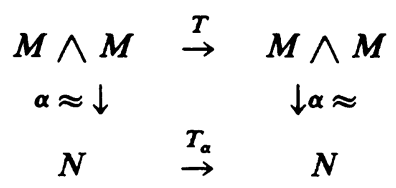

Proposition 20. There exists an $\alpha: M \wedge M \rightarrow N$ satisfying the conditions of Proposition 19(a) such that
(a) $T_{\alpha} j_{0}=j_{0}+j_{1} \pi: M \rightarrow N$,
(b) $k_{0} T_{\alpha}=k_{0}+i k_{1}: N \rightarrow S M$.

5. Definition of a compatible multiplication. We assume now, that if $q \equiv 2$ $(\bmod 4)$, then $\left[q_{M}\right]_{E}=0$ (Proposition 10). We also suppose we have chosen a fixed $\alpha: M \wedge M \rightarrow N$ satisfying Proposition 20. Our aim is to define then a proper multiplication, or equivalently, a multiplication generator.

From the definition of $N_{q}(16)$, it may be seen that we can regard $M_{q} \vee S^{1}$ as a subspectrum of $N_{q}$, where if $q \neq 2(\bmod 4)$, we regard $S^{1}$ as a subspectrum of $S M$ via $i: S^{1} \rightarrow S M$. Let $i_{0}: M_{q} \vee S^{1} \rightarrow N_{q}$ denote the inclusion. Then
(a) $i_{0} n_{1}=j_{0}: M \rightarrow N$,
(b) $i_{0} n_{2}=j_{1}: S^{1} \rightarrow M$.

Proposition 21. $A$ morphism $\tau: M \wedge M \rightarrow E \wedge M$ is a multiplication generator if and only if $\tau=\omega \alpha$ for some $\omega: N \rightarrow E \wedge M$ with $\omega i_{0}=(L \wedge 1) p_{1}$ : $M \vee S^{1} \rightarrow E \wedge M$

Proof. See [8, pp. 37-38].

Proposition 22. If $q \neq 2(\bmod 4)$ or $q \equiv 2(\bmod 4)$ and $\left[q_{M}\right]_{E}=0$, then there exists a multiplication generator $\tau: M \wedge M \rightarrow E \wedge M$.

Proof. We must find $\omega: N \rightarrow E \wedge M$ satisfying the condition of Proposition 21. If $q \neq 2(\bmod 4)$, we simply let $\omega=(L \wedge 1) p_{1}: N=M \vee S M \rightarrow E$ $\wedge M$. If $q \equiv 2(\bmod 4)$, the problem is to extend $(L \wedge 1) p_{1}: M \vee S^{1} \rightarrow E \wedge$ $M$ to $N=\left(M_{q} \vee S^{1}\right) U \gamma e^{2}$. If we consider representative spaces, we see that an extension to the stable 2-cell corresponds to a homotopy of $(L \wedge 1)$ in to 0 . Thus such an extension exists if (and only if) $[i \eta]_{E}=0$. But this condition is equivalent to $\left[q_{M}\right]_{E}=0$ by Lemma 5 and Proposition 13.

THEOREM 23. (a) If $q \equiv 2(\bmod 4)$ there exists a compatible multiplication $\mu_{q}$ on $E \wedge M$, which is proper and is quasi-associative if $\mu$ is associative $(\bmod q)$.

(b) If $q \equiv 2(\bmod 4)$ there exists a compatible multiplication $\mu_{q}$ on $E \wedge M$ if and only if the conditions of Proposition 13 are satisfied. If these conditions hold $\mu_{q}$ may be chosen to be proper and if $\mu$ is commutative $(\bmod q)$ and associative $(\bmod q)$, then $\mu_{q}$ may be chosen to be quasi-associative.

Proof. The existence of a proper $\mu_{q}$ follows from Proposition 16(b) provided $\mu$ is both commutative $(\bmod q)$ and associative $(\bmod q)$. If $q \neq 2$ 
(mod 4) then from the proof of Proposition 22 it is clear that the chosen multiplication generator $\tau$ has the form $(L \wedge 1) \tilde{\mu}_{q}: M \wedge M \rightarrow M \rightarrow E \wedge M$, where $\tilde{\mu}_{q}$ is a multiplication on $M$. Given this, it may be checked that Proposition 16(b), showing $\mu_{q}$ to be quasi-associative, holds without the assumption of commutativity $(\bmod q)$.

If we examine the proof of Proposition 22 , we see that if $q \equiv 2(\bmod 4)$, there is an arbitrary choice (of an extension) involved in the construction of $\tau$. Our $\mu_{q}$ in this case is thus not canonical. If $q \neq 2(\bmod 4)$, however, $\tau$ and $\mu_{q}$ are specifically determined, given a fixed $\alpha$. If $q$ is odd, then by Proposition 19(c) $\alpha$ is unique. Thus in this case we have a canonical choice for $\mu_{q}$. If $q \equiv 0$ (mod 4) then by Proposition 19(b) and the proof of Proposition 20 it may be seen that there are exactly two choices for $\alpha$ which satisfy Proposition 19(a) and Proposition 20. If those are designated $\alpha$ and $\alpha^{\prime}$, they are related by $\alpha^{\prime}=\alpha\left(1+j_{0} i \eta^{2} \pi k_{0}\right) \alpha$. Thus in this case we have two canonical choices of $\mu_{q}$ with no distinction between them.

\section{PART III: AdDitional Properties}

1. Bockstein multiplications. We consider now the existence of compatible multiplications having the various additional properties previously discussed. In this section we consider the existence of compatible multiplications which are Bockstein.

We continue to assume we have fixed $\alpha$ satisfying Propositions 19(a) and 20.

ProposItion 24. If $q \neq 2(\bmod 4)$ the multiplication $\mu_{q}$ on $E \wedge M$ of Theorem 23 is Bockstein.

Proof. This $\mu_{q}$ is derived from a multiplication $\tilde{\mu}_{q}$ on $M$. It will suffice to show that $\tilde{\mu}_{q}=p_{1} \alpha: M \wedge M \rightarrow N=M \vee S M \rightarrow M$ is Bockstein. To do this we use the condition of Lemma 3. Using the results of \$II.4 we have

$$
\begin{aligned}
\gamma_{1}+\gamma_{2} & =(1 \wedge \pi)+(1 \wedge \pi) T=\left(k_{0}+k_{0} T_{\alpha}\right) \alpha \\
& =\left(k_{0}-k_{0}+i k_{1}\right) \alpha=i k_{1} \alpha=i \pi p_{1} \alpha=\beta \mu_{q}
\end{aligned}
$$

as desired.

COROLlary 25. If $q \neq 2(\bmod 4)$ and $\mu$ is associative $(\bmod q)$ then there is a quasi-associative Bockstein multiplication $\mu_{q}$ on $E \wedge M$.

Proof. This follows from the above and Theorem 23(a).

Proposition 26. If $q \equiv 2(\bmod 4)$ there exists a compatible multiplication on $E \wedge M$ which is Bockstein if and only if $[\eta]_{E}=0$.

Proof. See [8, pp. 40-41]. The proof is ultimately analogous to that of Proposition 22. 
COROLLARY 27. If $q \equiv 2(\bmod 4)$ and $\mu$ is associative $(\bmod q)$ and commutative $(\bmod q)$, then $[\eta]_{E}=0$ is a necessary and sufficient condition for the existence of a quasi-associative Bockstein multiplication on $E \wedge M$.

2. Commutativity and associativity when $q \neq 2(\bmod 4)$.

LEMMA 28. If $q \neq 2(\bmod 4)$ then

$$
T_{\alpha}=n_{1} p_{1}+n_{2} \delta p_{2}-n_{2} p_{2}+n_{1}\left(\varepsilon i \eta^{2} \pi\right) p_{2}: N=M \vee S M \rightarrow N=M \vee S M
$$

where $\varepsilon=0$ if $q$ is odd and either $\varepsilon=0$ or $\varepsilon=1$ if $q \equiv 0(\bmod 4)$. (Recall that $\delta=(i \wedge 1) \pi: M \rightarrow S M$. $)$

Proof. Since the wedge product is both a sum and a product in our category, $T_{\alpha}$ is clearly the sum of four component morphisms of the type given. The first three components, as expressed in the lemma, are determined by Proposition 20. The fourth component must have the form $n_{1} \lambda p_{2}$ for some $\lambda: S M \rightarrow M$. By (11), $\lambda=0$ if $q$ is odd, while if $q \equiv 0(\bmod 4)$ then $\lambda=\varepsilon i \eta^{2}+\varepsilon_{1} \tilde{\eta} \pi+\varepsilon_{2} i \bar{\eta}$ where each of $\varepsilon, \varepsilon_{1}$ and $\varepsilon_{2}$ is either 0 or 1 . We also know that $\left(T_{M, M}\right)^{2}=1$ and thus by (19) $T_{\alpha}^{2}=1$. $T_{\alpha}$ can be computed directly in terms of $\varepsilon, \varepsilon_{1}$, and $\varepsilon_{2}$, and it may be checked that

$$
\begin{aligned}
T_{\alpha}^{2} & =n_{1} p_{1}+n_{2} p_{2}+n_{1}\left(\varepsilon_{2} i \eta \pi\right) p_{1}+n_{2}\left(\varepsilon_{1} i \eta \pi\right) p_{2} \\
& =1_{N}+n_{1}\left(\varepsilon_{2} i \eta \pi\right) p_{1}+n_{2}\left(\varepsilon_{1} i \eta \pi\right) p_{2} .
\end{aligned}
$$

From this we conclude $\varepsilon_{1}=\varepsilon_{2}=0$.

Proposimon 29. Suppose $\mu$ is commutative $(\bmod q)$. Then if $q$ is odd the multiplication of Theorem 23 is commutative, while if $q \equiv 0(\bmod 4)$ a sufficient condition for this multiplication to be commutative is $\left[\eta^{2} \pi\right]_{E}=0$ or equivalently $\left[i^{2}\right]_{E}=0$.

Proof. $\mu_{q}$ is generated by $\tau_{q}=(L \wedge 1) p_{1} \alpha$, and is commutative if $\tau_{q}$ is. Now

$$
\begin{aligned}
\tau_{q} T & =(L \wedge 1) p_{1} T_{\alpha} \alpha=(L \wedge 1) p_{1}\left(n_{1} p_{1}+n_{2} \delta p_{1}-n_{2} p_{2}+n_{1}\left(\varepsilon i \eta^{2} \pi\right) p_{2}\right) \alpha \\
& =(L \wedge 1) p_{1} \alpha+\varepsilon(L \wedge 1) i \eta^{2} p_{2} \alpha=\tau_{q}+\varepsilon(L \wedge 1) i \eta^{2} \pi p_{2} \alpha .
\end{aligned}
$$

Thus if $q$ is odd (and hence $\varepsilon=0$ ) or if $\left[i \eta^{2} \pi\right]_{E}=0$ then $\tau_{q} T=\tau_{q}$. But exactly analogously to the proof of Proposition $13,\left[i \eta^{2} \pi\right]_{E}=0$ is equivalent to $\left[i \eta^{2}\right]_{E}=0$ or $\left[\eta^{2} \pi\right]_{E}=0$ (or analogues of the three other conditions of Proposition 13).

By technical arguments, this result can be strengthened slightly.

Proposition 30. If $\mu$ is commutative $(\bmod q)$ and $q \equiv 0(\bmod 4)$ and $\left[i \eta^{2} \pi\right]_{E}=2 \lambda$ for some $\lambda: S M \rightarrow E \wedge M$ then there is a proper commutative multiplication $\mu_{q}^{\prime}$ on $E \wedge M$ which is also Bockstein. If $\mu$ is also associative $(\bmod q), \mu_{q}^{\prime}$ is quasi-associative. 
Proof. See [8, p. 43].

It is not hard to verify that if $q \equiv 0(\bmod 4)$ and $\varepsilon=1$, the condition of Proposition 30 is also necessary to have a commutative compatible multiplication, while if $\varepsilon=0$ the multiplication of Theorem 23 is always commutative. Thus given $\varepsilon$, we have a necessary and sufficient condition for a commutative multiplication. As previously noted, when $q \equiv 0(\bmod 4)$ there are two choices for $\alpha$ satisfying Propositions 19(a) and 20. It is easily checked, however, that $\varepsilon$ is independent of $\alpha$. Unfortunately, it does not appear easy to determine $\varepsilon$.

We now turn to associativity.

Proposirion 31. If $q \neq 2(\bmod 4), \mu$ is associative $(\bmod q)$, and $[\tilde{\eta} \eta]_{E}=0$ $=[i \nu]_{E}$, then the multiplication of Theorem 23 is associative.

Proof. See [8, pp. 44-45].

The conditions of Proposition 31 may, of course, be replaced by the Spanier-Whitehead dual conditions $[\eta \bar{\eta}]_{E}=0$ and $[\nu \pi]_{E}=0$ if desired, or by other equivalent conditions analogous to those of Proposition 13. The following result summarizes what we have shown when $q \neq 2(\bmod 4)$, except that it does not include Proposition 30.

THEOREM 32. If $q \neq 2(\bmod 4)$ there exists a quasi-associative Bockstein compatible multiplication $\mu_{q}$ on $E \wedge M . \mu_{q}$ is commutative if $\mu$ is commutative $(\bmod q)$ and $q$ is odd, or if $\left[i^{2}\right]_{E}=0 . \mu_{q}$ is commutative if $\mu$ is commutative $(\bmod q)$ and $q$ is odd, or if $\left[i \eta^{2}\right]_{E}=0 . \mu_{q}$ is associative if $\mu$ is associative $(\bmod q)$ and $q$ is relatively prime to 2 or 3 , or if $q$ is odd and $[i v]_{E}=0$, or if $[\tilde{\eta} \eta]_{E}=[i \nu]_{E}=0$.

3. Reduction to prime powers. This section may be compared with [3, pp. 94-95]. Let $r, s \geqslant 2$ be relatively prime integers with $s$ odd.

Notice that $M_{r} \wedge M_{s}$ is trivial since its stable order must divide both $2 r$ and $s$, and thus is 1 . Also $\left[M_{r}, M_{s}\right]=\left[M_{s}, M_{r}\right]=0$ since $M_{r}$ and $M_{s}$ have relatively prime stable orders. It is clear as well that $M_{r} \vee M_{s}$ is equivalent to $M_{r s}$ since the two spectra have the same integral homology groups. We shall find morphisms which essentially determine such an equivalence.

Choose integers $c$ and $d$ such that $c r+d s=1$. Define $A: M_{r s} \rightarrow M_{r} \vee M_{s}$ by $A=n_{1} \bar{s}_{r, s}+n_{2} \bar{r}_{s, r}$ and $B: M_{r} \vee M_{s} \rightarrow M_{r s}$ by $B=d \overline{1}_{r, s} p_{1}+c \overline{1}_{s, r} p_{2}$. We shall drop the subscripts on $\overline{1}, \bar{r}$ and $\bar{s}$ henceforth.

LEMMA 33. If $r$ is odd (as well as s) then $B A=1_{M_{r}}$ and $A B=1_{M_{r} \vee M_{s}}$. If $r$ is even and $\left[i_{r s} \eta \pi\right]_{E}=0$ then $[A B]_{E}=\left[1_{M_{r s}}\right]_{E}$ and $[B A]_{E}=\left[1_{M_{r} \vee M_{s}}\right]_{E}$.

THEOREM 34. If $r$ is odd or if $r$ is even and $\left[i_{r s} \eta \pi_{r}\right]_{E}=0$, then there is a bijection between multiplication generators $\tau_{r s}: M_{r s} \wedge M_{r s} \rightarrow E \wedge M_{r s}$ and pairs 
$\left(\tau_{r}, \tau_{s}\right)$ of multiplication generators $\tau_{r}: M_{r} \wedge M_{r} \rightarrow E \wedge M_{r}$ and $\tau_{s}: M_{s} \wedge M_{s} \rightarrow$ $E \wedge M_{s}$. Under this correspondence $\tau_{r s}$ is commutative (associative, Bockstein) if and only if both $\tau_{r}$ and $\tau_{s}$ are commutative (associative, Bockstein).

Theorem 34 gives a correspondence for multiplication generators, that is, for morphisms defined on $M \wedge M$. A similar correspondence holds for compatible multiplications, that is, for morphisms defined on $E \wedge M \wedge E \wedge$ $M$. Thus if $[\eta]_{E}=0$ (and thus Theorem 34 applies), then our whole general problem may be regarded as reduced to the case where $q$ is a prime power. We shall use this to reduce the problem of determining the existence of commutative and associative multiplications when $q \equiv 2(\bmod 4)$ to the case of $q=2$.

4. Commutativity when $q \equiv 2(\bmod 4)$. As mentioned previously, we shall consider first the case of $q=2$. Let $\alpha: M_{2} \wedge M_{2} \rightarrow N_{2}$ satisfy Propositions 19(a) and 20. We shall need information about $T_{\alpha}$. For this purpose we need to analyse the group $\left[N_{2}, N_{2}\right]$. This section is similar to [4, pp. 85-88].

LEMMA 35. In [SM,$\left.M_{2}\right], 1 \wedge \eta=\tilde{\eta} \pi+i \bar{\eta}$.

Proposition 36. $\left[N_{2}, N_{2}\right]=Z_{4} \oplus Z_{4} \oplus Z_{2}$ with generators $1, j_{1} k_{1}$ and $j_{0} i \bar{\eta} k_{0}$.

Proposition 37. $T_{\alpha}=1_{N}+j_{1} k_{1}+j_{0} i \bar{\eta} k_{0}(\bmod 2[N, N])$.

Proposition 38. If $q=2$ and $\mu$ is commutative $(\bmod q)$ then there is a commutative compatible multiplication $\mu_{q}$ on $E \wedge M$ if and only if $[\bar{\eta}]_{E}=0$. If this condition holds $\mu_{q}$ may be chosen to be Bockstein and, if $\mu$ is also associative $(\bmod q)$, to be quasi-associative.

Proof. See [8, pp. 51-52].

Note that by Lemma 35 we could replace the condition $[\bar{\eta}]_{E}=0$ by $[\tilde{\eta}]_{E}=0$.

COROLlary 39. Proposition 38 holds with $q=2$ replaced by $q \equiv 2(\bmod 4)$.

Proof. This follows from Propositions 38 and 29 and Theorem 34.

5. Associativity when $q \equiv 2(\bmod 4)$. Again we consider the case of $q=2$. Fix $\alpha: M_{2} \wedge M_{2} \rightarrow M_{2}$ as in the previous section. Let $\lambda_{1}$ and $\lambda_{2}$ denote morphisms making the following diagrams commute.

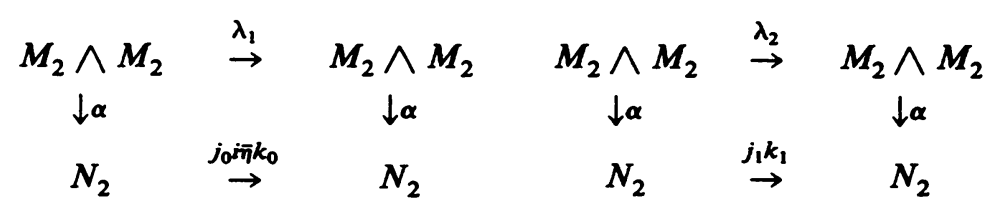


$\lambda_{1}$ can be defined explicitly as $(i \wedge i) \bar{\eta}(1 \wedge \pi)$. By Proposition 37 then

$$
T_{M_{2}, M_{2}}=1_{M_{2}}+\lambda_{1}+\lambda_{2}\left(\bmod 2\left[M_{2} \wedge M_{2}, M_{2} \wedge M_{2}\right]\right) \text {. }
$$

Proposition 40. If $q=2,[\eta]_{E}=0$ and $\mu$ is commutative $(\bmod q)$ and associative $(\bmod q)$, then there is an associative Bockstein multiplication $\mu_{q}$ on $E \wedge M_{q}$. If $[\bar{\eta}]_{E}=0$, then this $\mu_{q}$ is also commutative.

Proof. The proof is long, though considerably simpler than [4, pp. 92-104], which achieves a similar result working in the category of spaces. Using Proposition 16, it suffices to work with multiplication generators. By Proposition 26 there is a multiplication generator $\tau$ which is Bockstein. We shall show that such a $\tau$ is associative. The final assertion of the Proposition will then follow from Proposition 38 and its proof.

Let $\psi$ denote the fixed equivalence $1 \wedge T: M \wedge M \wedge M \rightarrow M \wedge M \wedge M$. It will be convenient to make use of $\psi$, as it takes the subspectrum $M \wedge S^{0} \wedge$ $M$ of $M \wedge M \wedge M$ to the subspectrum $M \wedge M \wedge S^{0}$, which is easier to deal with. We want to show (see (14)) that $\tau_{L}=\tau_{R}$. It can be checked that $\tau_{R}$ is equivalent to the following composition.

$$
\begin{aligned}
& M \wedge M \wedge M^{\psi(T \wedge 1) \psi \equiv \underset{\psi\left(1+\lambda_{1}+\lambda_{2}\right) \psi}{\rightarrow}} M \wedge M \wedge M \\
& \stackrel{T \wedge 1 \equiv\left(1+\lambda_{1}+\lambda_{2}\right) \wedge 1}{\rightarrow} M \wedge M \wedge M \stackrel{\tau \wedge 1}{\rightarrow} E \wedge M \wedge M
\end{aligned}
$$

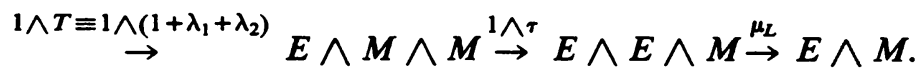

Note that the first morphism $\psi(T \wedge 1) \psi$ switches the first and third factors in $M \wedge M \wedge M$.

For the three morphisms involving $T_{M, M}$ in (20) we have indicated $T \equiv 1$ $+\lambda_{1}+\lambda_{2}$. This of course is $\bmod 2[M \wedge M, M \wedge M]$. As far as the total composition is concerned, since $\left[2_{M}\right]_{E}=0$, these congruences may be regarded as equalities. Also, by Lemma $35, \lambda_{1}=(i \wedge i) \bar{\eta}(1 \wedge \pi)$ may be replaced by $(1 \wedge i) \tilde{\eta}(\pi \wedge \pi)$ if desired, since $[\eta]_{E}=0$. Thus (20) may be regarded as a sum of twenty-seven morphisms. By the way $\tau$ is defined, however (essentially by Proposition 21 ), $\tau \lambda_{2}=0$. Thus we need not consider the $\lambda_{2}$ involved in the second two terms involving $T$ in (25). Thus we are left with a sum of twelve morphisms, which may be described as morphisms of the form $(x, y, z)$ where $x$ may be $1, \lambda_{1}$, or $\lambda_{2}$, and $y$ and $z$ may each be either 1 or $\lambda_{1}$.

Now it may easily be checked that the $(1,1,1)$ morphism is $\tau_{L}$. Thus it remains to show that the sum of the other eleven morphisms is trivial. For the details of this, see [8, pp. 54-56]. 
COROLlaRY 41. Proposition 40 holds with $q=2$ replaced by $q \equiv 2(\bmod 4)$ with the additional hypothesis in case $q \equiv 0(\bmod 3)$ that $[i v]_{E}=0$.

Proof. Using Theorem 34, this follows from Proposition 40 and Theorem 36 , except that when $q$ is a multiple of three we must assume $\left[i_{q / 2} \nu\right]_{E}=0$. However, since $i_{q / 2}=\overline{2} i_{q}: S^{0} \rightarrow M_{q} \rightarrow M_{q / 2}$ by (8)(a), it follows that [iv] $]_{E}=$ 0 , i.e., $\left[i_{q} \nu\right]_{E}=0$, is sufficient.

\section{Part IV: Classification of Multiplications}

1. General classifications. The general idea of this part is as follows. Suppose $\mu_{q}$ is a compatible multiplication on $E \wedge M$ having certain properties. Then we want to classify relative to $\mu_{q}$, in a sense to be made precise, all compatible multiplications on $E \wedge M$. We shall also classify multiplication generators, which is equivalent, essentially by Proposition 17, to classifying proper multiplications.

Proposition 42. If there exist any multiplication generators $\tau: E \wedge M \rightarrow E$ $\wedge M$, there is a one-to-one correspondence of the set of all such multiplication generators, or of the set of all proper multiplications on $E \wedge M$, with $\left[S^{2}\right.$, $E \wedge M] \approx h^{-2}\left(S^{0} ; Z_{q}\right)$. If $d\left(\tau^{\prime}, \tau\right)$ denotes the element of $\left[S^{2}, E \wedge M\right]$ corresponding to a multiplication generator $\tau^{\prime}$, then $\tau^{\prime}-\tau=d\left(\tau, \tau^{\prime}\right)(\pi \wedge \pi): M \wedge$ $M \rightarrow E \wedge M$.

Proof. We may regard $M \wedge S^{0}$ and $S^{0} \wedge M$ as subspectra of $M \wedge M$ with inclusions $1 \wedge i$ and $i \wedge 1$ respectively. Let $\bar{M}$ denote the union of these subspectra and $\bar{i}: M \rightarrow M \wedge M$ its inclusion. Considering the stable cell structure of $M \wedge M$ we have a cofiber sequence

$$
M \stackrel{\bar{i}}{\rightarrow} M \wedge M \stackrel{\bar{\pi}}{\rightarrow} S^{2} .
$$

By Proposition 16(a) a morphism $\tau^{\prime}: M \wedge M \rightarrow E \wedge M$ is a multiplication generator if and only if $\tau^{\prime} \bar{i}$ is as specified by (15). The classification claimed for multiplication generators then follows from the cofiber sequence (21). By Corollary 18 there is a correspondence of multiplication generators with proper multiplications, so proper multiplications are classified in the same way.

Proposition 42 defines $d\left(\tau^{\prime}, \tau\right) \in\left[S^{2}, E \wedge M\right]$ for multiplication generators $\tau$ and $\tau^{\prime}$. Let us also let $d\left(\mu_{q}^{\prime}, \mu_{q}\right)=d\left(\tau\left(\mu_{q}^{\prime}\right), \tau\left(\mu_{q}\right)\right)$ for proper multiplications $\mu_{q}$ and $\mu_{q}^{\prime}$.

Proposirion 43. (a) Let $\mu_{q}: E \wedge M \wedge E \wedge M \rightarrow E \wedge M$ be a compatible multiplication. Then there is a one-to-one correspondence of the set of all such compatible multiplications with $\left[E \wedge S^{1} \wedge E \wedge S^{1}, E \wedge M\right] \approx h^{-2}(E \wedge$ $\left.E ; Z_{q}\right)$. If $e\left(\mu_{q}^{\prime}, \mu_{q}\right)$ denotes the element of $\left[E \wedge S^{1} \wedge E \wedge S^{1}, E \wedge M\right]$ corresponding to a compatible multiplication $\mu_{q}^{\prime}$, then 


$$
\mu_{q}^{\prime}-\mu_{q}=e\left(\mu_{q}^{\prime}, \mu_{q}\right)(1 \wedge \pi \wedge 1 \wedge \pi): E \wedge M \wedge E \wedge M \rightarrow E \wedge M .
$$

(b) If $\mu_{q}$ is proper then the proper multiplications correspond to the subgroup of $\left[E \wedge S^{1} \wedge E \wedge S^{1}, E \wedge M\right]$ consisting of $\lambda$ of the form $\mu_{L}(\mu \wedge \theta)(1 \wedge T \wedge$ 1) for some $\theta \in\left[S^{2}, E \wedge M\right]$. If $\mu_{q}$ corresponds to such a $\lambda$ then $d\left(\mu_{q}^{\prime}, \mu_{q}\right)=(L$ $\wedge 1 \wedge\llcorner\wedge 1) e\left(\mu_{q}^{\prime}, \mu_{q}\right)$.

Proof. (a) is proved in the same manner as Proposition 42 regarding $E \wedge M \wedge E \wedge S^{0}$ and $E \wedge S^{0} \wedge E \wedge M$ as subspectra of $E \wedge M \wedge E \wedge$ $M$ with respective inclusions $1 \wedge 1 \wedge 1 \wedge i$ and $1 \wedge i \wedge 1 \wedge 1$.

(b) then follows from the definition of proper multiplications and Proposition 42.

Define $C:\left[S^{2}, E \wedge M\right] \rightarrow\left[E \wedge S^{1} \wedge E \wedge S^{1}, E \wedge M\right]$ by $C(\theta)=\mu_{L}(\mu$ $\wedge \theta)(1 \wedge T \wedge 1)$ as in Proposition 43(b). Then $C$ is injective, as $\theta=\lambda(L \wedge 1$ $\wedge L \wedge 1)$. The two preceding propositions can then also be expressed in the following manner.

COROLlary 44. If there exists compatible multiplications on $E \wedge M$ (i.e., if $q \equiv 2(\bmod 4)$ or if $\left.[q]_{E}=0\right)$, then the group $\left[E \wedge S^{1} \wedge E \wedge S^{1}, E \wedge M\right]$ acts transitively without fixed points on the set of such compatible multiplications. Regarding $\left[S^{2}, E \wedge M\right]$ as a subgroup under $C$, it acts transitively on the proper multiplications.

2. Quasi-associativity. By Proposition 16(b) we know that if $\mu$ is commutative $(\bmod q)$ and associative $(\bmod q)$, then every proper multiplication is quasi-associative. The converse also holds.

Proposition 45. If $\mu$ is commutative $(\bmod q)$ and associative $(\bmod q)$ then a compatible multiplication on $E \wedge M$ is quasi-associative if and only if it is proper. Thus under these hypotheses we may replace "proper" by "compatible and quasi-associative" in Propositions 42 and 43, and Corollary 44.

Proof. As noted, the "if" part comes from Proposition 16(b). For the "only if' part, we shall show that if $\mu_{q}^{\prime}$ is quasi-associative, then $\mu_{q}^{\prime}=\mu\left(\tau\left(\mu_{q}^{\prime}\right)\right)$, and thus is proper. Let $\mu_{q}=\mu\left(\tau\left(\mu_{q}^{\prime}\right)\right)$. Then $\tau\left(\mu_{q}\right)=\tau\left(\mu_{q}^{\prime}\right)$. Thus

$$
0=d\left(\tau\left(\mu_{q}^{\prime}\right), \tau\left(\mu_{q}\right)\right)=e\left(\mu_{q}^{\prime}, \mu_{q}\right)(L \wedge 1 \wedge L \wedge 1) \text {. }
$$

It remains to show that $e\left(\mu_{q}^{\prime}, \mu_{q}\right)(L \wedge 1 \wedge L \wedge 1)=0$, together with the quasi-associativity of $\mu_{q}^{\prime}$ and $\mu_{q}$, implies $e\left(\mu_{q}^{\prime}, \mu_{q}\right)=0$, and thus $\mu_{q}^{\prime}=\mu_{q}$. For this see [8, pp. 59-60].

\section{Bockstein multiplications.}

Proposition 46. Suppose $\tau$ is a multiplication generator which is Bockstein. Then a multiplication generator $\tau^{\prime}$ is Bockstein if and only if $\beta d\left(\tau^{\prime}, \tau\right)=0$. Likewise if $\mu_{q}$ is proper and Bockstein and $\mu_{q}^{\prime}$ is proper, then $\mu_{q}^{\prime}$ is Bockstein if 
and only if $\beta d\left(\mu_{q}^{\prime}, \mu_{q}\right)=0$. If $\mu_{q}$ is any compatible multiplication, then $\mu_{q}^{\prime}$ is Bockstein if and only if $\beta e\left(\mu_{q}^{\prime}, \mu_{q}\right)=0$.

Proof. The idea is the same in all cases. For multiplication generators, $\tau^{\prime}$ is Bockstein if and only if $\beta \tau=\tilde{\gamma}_{1}+\tilde{\gamma}_{2}$. Now $\tilde{\gamma}_{1}$ and $\tilde{\gamma}_{2}$ do not depend on the choice of multiplication generators. Thus if $\tau$ is Bockstein, then $\tau^{\prime}$ is if and only if $0=\beta\left(\tau^{\prime}-\tau\right)=\beta d\left(\tau^{\prime}, \tau\right)(\pi \wedge \pi)$. By the usual exact sequence argument this is equivalent to $\beta d\left(\tau^{\prime} \tau\right)=0$.

COROLLARY 47. If there exist compatible multiplications on $E \wedge M$ which are Bockstein (i.e., if $q \equiv 2(\bmod 4)$ or $\left.[\eta]_{E}=0\right)$, then the group $\operatorname{ker}\left(\beta_{*}\right) \subset[E \wedge$ $\left.S^{1} \wedge E \wedge S^{1}, E \wedge M\right]$ acts transitively without fixed points on the set of all such. The subgroup $\operatorname{ker}\left(\beta_{*}\right) \subset\left[S^{2}, E \wedge M\right]$ (with inclusions induced by $C$ ) acts transitively on the proper multiplications which are Bockstein.

COROLlary 48. In Corollary 47 we may replace

$$
\operatorname{ker}\left(\beta_{*}\right) \subset\left[E \wedge S^{1} \wedge E \wedge S^{1}, E \wedge M\right]
$$

by

$$
\left[E \wedge S^{1} \wedge E \wedge S^{1}, E\right] \otimes Z_{q} \oplus \operatorname{Tor}\left(q\left[E \wedge S^{1} \wedge E, E\right], Z_{q}\right)
$$

and

$$
\operatorname{ker}\left(\beta_{*}\right) \subset\left[S^{2}, E \wedge M\right] \text { by }\left[S^{2}, E\right] \otimes Z_{q} \oplus \operatorname{Tor}\left(q\left[S^{1}, E\right], Z_{q}\right) .
$$

Proof. The two assertions are proved in the same manner. We prove the latter. If there exists compatible multiplications on $E \wedge M$ then by Proposition 7 we have a split short exact sequence

$$
\begin{array}{r}
0 \rightarrow\left[S^{2}, E\right] \otimes Z_{q} \stackrel{(1 \wedge i)}{\rightarrow}\left[S^{2}, E \wedge M\right] \stackrel{(1 \wedge \pi)}{\rightarrow} \operatorname{Tor}\left(\left[S^{2}, E \wedge S^{1}\right], Z_{q}\right) \rightarrow 0 \\
\prod \\
\operatorname{Tor}\left(\left[S^{1}, E\right], Z_{q}\right)
\end{array}
$$

Given $\theta \in\left[S^{2}, E \wedge M\right]$ we have $\beta(\theta)=(1 \wedge i)(1 \wedge \pi) \theta$. By (1) then, $\beta(\theta)$ $=0$ if and only if $(1 \wedge \pi) \theta$ is a multiple of $q$ in $\left[S^{2}, E \wedge S^{1}\right] \approx\left[S^{1}, E\right]$. The desired conclusion then follows from the splitting of (22).

Roughly, $\operatorname{ker}\left(\beta_{*}\right)$ classifies compatible multiplications (proper multiplications, multiplication generators) which are Bockstein in the various cases. $\mathbf{A}$ subgroup of $\operatorname{ker}\left(\beta_{*}\right) \subset\left[S^{1}, E \wedge M\right]$ is $\operatorname{ker}\left((1 \wedge \pi)_{*}\right)$. By (1) this is $\operatorname{Im}((1 \wedge$ $\left.i)_{*}\right):\left[S^{2}, E\right] \rightarrow\left[S^{2}, E \wedge M\right]$ and thus is isomorphic to $\left[S^{2}, E\right] \otimes Z_{q}$ or $h^{-2}(E) \otimes Z_{q}$. This corresponds to the first summand in the expression given in Corollary 48. Thus if $\mu_{q}$ is proper and Bockstein then a subset of the set of all compatible multiplications with these properties is in one-one correspondence with $h^{-2}(E) \otimes Z_{q}$. We shall discuss this subset in IV.5. 


\section{Commutativity.}

ProposIrIon 49. Suppose $\tau$ is a commutative multiplication generator. Then a multiplication generator $\tau^{\prime}$ is commutative if and only if $2 d\left(\tau^{\prime}, \tau\right)=0$. A similar assertion holds with "multiplication generator" replaced by "proper multiplication".

Proof. Let $\alpha: M \wedge M \rightarrow N$ satisfy Propositions 19(a) and 20. Since $\tau$ is commutative, $\tau^{\prime}$ is commutative if and only if $\left(\tau^{\prime}-\tau\right) T_{M, M}=\tau^{\prime}-\tau$. We have $\tau^{\prime}-\tau=d\left(\tau^{\prime}, \tau\right)(\pi \wedge \pi)$ and

$$
\begin{aligned}
\left(\tau^{\prime}-\tau\right) T_{M, M} & =d\left(\tau^{\prime}, \tau\right)(\pi \wedge 1)(1 \wedge \pi) T_{M, M}=d\left(\tau^{\prime}, \tau\right)(\pi \wedge 1) k_{0} \alpha T_{M, M} \\
& =d\left(\tau^{\prime}, \tau\right)(\pi \wedge 1) k_{0} T_{\alpha} \alpha=d\left(\tau^{\prime}, \tau\right)(\pi \wedge 1)\left(-k_{0}+i k_{1}\right) \alpha \\
& =-d\left(\tau^{\prime}, \tau\right)(\pi \wedge 1) k_{0} \alpha=-d\left(\tau^{\prime}, \tau\right)(\pi \wedge \pi)
\end{aligned}
$$

Thus $\tau^{\prime}$ is commutative if and only if $d\left(\tau^{\prime}, \tau\right)(\pi \wedge \pi)=-d\left(\tau^{\prime}, \tau\right)(\pi \wedge \pi)$. By an exact sequence argument based on (1), this is equivalent to $2 d\left(\tau^{\prime}, \tau\right)=$ 0 . The second assertion of the proposition is, of course, equivalent to the first.

COROLLARY 50. If there exist commutative multiplication generators (commutative proper multiplications, commutative quasi-associative compatible multiplications), then $\operatorname{Tor}\left(\left[S^{2}, E \wedge M\right], Z_{2}\right)$ acts transitively without fixed points on the set of all such. If one of these is Bockstein, then the subgroup $\operatorname{ker}\left(\beta_{*}\right)$ of this group acts transitively on the set of all such.

COROLlaRY 51. If $q$ is odd, commutative multiplication generators (commutative proper multiplications, commutative quasi-associative compatible multiplications if $\mu$ is commutative $(\bmod q)$ and associative $(\bmod q))$ exist and are unique.

COROLLARY 52. For $q=2$, if there is a commutative multiplication generator (proper multiplication, quasi-associative compatible multiplication), i.e., if $[\bar{\eta}]_{E}$ $=0($ and $\mu$ is commutative $(\bmod q)$ and associative $(\bmod q)$ for the compatible multiplication case), then all multiplication generators (proper multiplications, quasi-associative compatible multiplications) are commutative.

5. Associativity. Recall that in $\$ 3$ we remarked that a subset of the set of proper multiplications which are Bockstein are in one-one correspondence with $h^{-2}(E) \otimes Z_{q}$.

Proposmion 53. If $\mu$ is commutative $(\bmod q)$ and $\mu_{q}$ is proper and Bockstein and is associative, then all of the multiplications corresponding to $h^{-2}(E) \otimes Z_{q}$ are associative. 
Proof. See [8, pp. 64-65].

6. Applications. We consider some applications of our results to some of the better known multiplicative cohomology theories. These theories (and their representing spectra) are stable cohomotopy $\left(S^{0}\right)$, ordinary cohomology $(K Z)$, complex $K$-theory $(B U)$, connective complex $K$-theory (bu), real $K$-theory $(B O)$, connective real $K$-theory (bo), complex cobordism $(M U)$, special unitary cobordism (MSU), and symplectic cobordism (MSp). Each has a standard multiplication which is commutative and associative.

Proposimon 54. There is a unique compatible multiplication on $S^{0} \wedge M \approx$ $M$ when $q$ is odd, which is Bockstein, commutative, and associative when $q$ is not a multiple of three. When $q \equiv 0(\bmod 4)$ there are two compatible multiplications on $M$ which are Bockstein. When $q \equiv 2(\bmod 4)$ there are no compatible multiplications on $M$.

Proof. This has essentially been shown previously.

Proposition 55. There is a unique quasi-associative compatible multiplication on $K Z \wedge M$ for all $q$, which is Bockstein, commutative and associative.

Proof. This is easily checked using our previous results and the fact that

$$
\left[S^{i}, K Z\right]= \begin{cases}Z, & i=0 \\ 0, & i \neq 0\end{cases}
$$

ProposIrIon 56. There are exactly $q$ quasi-associative compatible multiplications on $B U \wedge M$. All are Bockstein and associative. Exactly one, two, or none of these are commutative, depending on whether $q$ is odd, $q \equiv 0(\bmod 4)$, or $q \equiv 2(\bmod 4)$

Proof. The homotopy groups of $B U$ are given by the periodicity theorem. From these it follows that $[\eta]_{B U}=0$ and $[\nu]_{B U}=0$. Thus by Theorem 32 and Corollary 41 there is at least one associative Bockstein compatible multiplication for all $q$. We have a short exact sequence

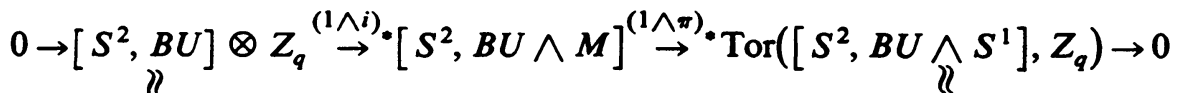

$$
\begin{aligned}
& Z_{q} Z_{Z^{\prime}} \quad \operatorname{Tor}\left(\left[S^{1}, B U\right], Z_{q}\right)
\end{aligned}
$$

Thus $\left[S^{2}, B U \wedge M\right] \approx Z_{q}$. Thus there are $q$ quasi-associative compatible multiplications by Propositions 42 and 45. By Proposition 46 they are all Bockstein, and by Proposition 53 they are all associative. By Corollary 51, when $q$ is odd there is always a unique commutative one. When $q \equiv 0$ $(\bmod 4)$ there is a commutative one by Proposition 29, and by Corollary 50 there are exactly two such. When $q \equiv 2(\bmod 4)[\bar{\eta}]_{B U} \neq 0$ by $[3$, pp. 83-84], 
and thus, by Proposition 38, there are no commutative compatible multiplications.

Proposition 57. The conclusions of Proposition 56 hold also for multiplications on $M U \wedge M$ and $b u \wedge M$.

Proof. There is a morphism $\psi: M U \rightarrow B U$ of ring spectra. This is discussed in [5]. Furthermore (see [5]), $\pi_{*}(M U)$ is a polynomial algebra over $Z$ with a generator in each even positive dimension. Thus $\left[S^{1}, M U\right] \approx 0$ and $\left[S^{2}, M U\right] \approx Z$. We have a commutative diagram

$$
\begin{array}{ccc}
{\left[S^{0}, M U\right]} & \stackrel{\eta^{*}}{\rightarrow} & {[S M, M U]} \\
\downarrow \psi_{*} & & \downarrow \psi_{*} \\
{\left[S^{0}, B U\right]} & \stackrel{\bar{\eta}^{*}}{\rightarrow} & {[S M, B U]}
\end{array}
$$

$\psi_{*}:\left[S^{0}, M U\right] \rightarrow\left[S^{0}, B U\right]$ is an isomorphism since $\psi$ is a morphism of ring spectra. Thus since $[\bar{\eta}]_{B U} \neq 0$ it follows that $[\bar{\eta}]_{M U} \neq 0$. Thus the proof of the preceding proposition applies equally well here.

As for $b u$, there is a morphism of ring spectra $b u \rightarrow B U$ which is an isomorphism on homotopy groups in nonnegative dimensions, so the same reasoning works in this case also.

Proposition 58. If $q$ is odd there is a unique quasi-associative compatible multiplication on $B O \wedge M$, which is Bockstein, commutative, and associative. If $q \equiv 0(\bmod 4)$ there are precisely four quasi-associative compatible multiplications on $B O \wedge M$, precisely two of which are Bockstein. If $q \equiv 2(\bmod 4)$ there are no compatible multiplications on $B O \wedge M$.

Proof. It is well known that $\left[S^{1}, B O\right] \approx Z_{2},\left[S^{2}, B O\right] \approx Z_{2}$, and $\left[S^{3}, B O\right]$ $\approx 0$. Thus $[\nu]_{B O}=0$. By Theorem 1.2 of $[1],[\eta]_{B O} \neq 0$. By an exact sequence argument based on (1), it follows that $[i \eta]_{B O} \neq 0$. Then by Proposition 13 there are no compatible multiplications on $B O \wedge M$ when $q \equiv 2(\bmod 4)$.

We have a short exact sequence

$$
\begin{gathered}
0 \rightarrow\left[S^{2}, B O\right] \otimes Z_{q} \stackrel{(1 \wedge i)}{\rightarrow}\left[S^{2}, B O \wedge M\right] \stackrel{(1 \wedge \pi)}{\rightarrow} * \operatorname{Tor}\left(\left[S^{2}, B O \wedge S^{1}\right], Z_{q}\right) \rightarrow 0 \\
\operatorname{Tor}\left(\left[S^{1}, B O\right], Z_{q}\right) .
\end{gathered}
$$

For $q$ odd we see that $\left[S^{2}, B O \wedge M\right] \approx 0$. By Theorem 32 and Propositions 42 and 45 we get the desired conclusion for $q$ odd. These same results and the short exact sequence imply that there are four quasi-associative compatible multiplications when $q \equiv 0(\bmod 4)$. By Proposition 46 and an exact sequence argument based on (1) we see that precisely two of these are Bockstein. 
Proposition 59. The conclusions of Proposition 58 hold also for multiplications on $M S U \wedge M, M S p \wedge M$ and bo $\wedge M$.

Proof. There are morphisms of ring spectra $M S p \rightarrow M S U \rightarrow B O$ (see [5]) and $b o \rightarrow B O$. By [6], $\left[S^{1}, M S U\right] \approx Z_{2} \approx\left[S^{2}, M S U\right]$ and $\left[S^{3}, M S U\right] \approx 0$, while by [7], $\left[S^{1}, M S p\right] \approx Z_{2} \approx\left[S^{2}, M S p\right]$ and $\left[S^{3}, M S p\right] \approx 0$. Using reasoning as in the proof of Proposition 57, the proof of Proposition 58 then goes through here.

Part V: The Bockstein Spectral Sequence

Let $p$ be a prime. Smashing (1) with $E$ we get a cofiber triangle

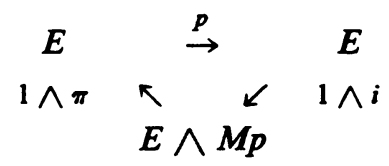

where $1 \wedge \pi$ has degree -1 .

For any $C W$-spectrum $X$ we then get an exact couple

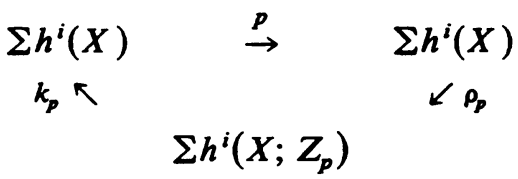

where $k_{p}$ is induced by $(1 \wedge \pi)$. The resulting spectral sequence $\left\{\Sigma_{i} E_{r}^{i}\left(X ; Z_{p}\right), r>1\right\}$ is the Bockstein spectral sequence of $X$ and $h$. Let $d_{r}$ denote the differential of $E_{r}$.

Additively this spectral sequence is quite analogous to the ordinary Bockstein spectral sequence. It converges if the coefficient groups of $h$ are finitely generated. We shall concern ourselves with obtaining multiplications on the successive $E_{r}^{*}$. This section may be compared with [4, pp. 108-118].

LEMMA 60. For integers $q, r>2$ there is a cofiber sequence

$$
\stackrel{i_{r} \pi_{q}}{\rightarrow} M_{r} \stackrel{\mathrm{I}}{\rightarrow} M_{r q} \stackrel{\text { I }}{\rightarrow} M_{q} \stackrel{i_{r} \pi_{q}}{\rightarrow}
$$

LEMMA 61. A morphism $\chi: X \rightarrow E \wedge M_{q}$ (of arbitrary degree) represents a class in $E_{r}^{*}\left(X ; Z_{p}\right)$ if and only if there is a commutative diagram

$$
\begin{array}{lll}
\quad E \wedge M & \searrow \overline{p^{r-1}} \\
X & \stackrel{x}{\rightarrow} & E \wedge M_{p}
\end{array}
$$

Proof. The proof is by induction on $r$ and is obvious for $r=1$. Suppose the lemma holds for $r$. Since by (9)(a), $\overline{p^{r}}=\overline{p^{r-1}} \bar{p}: M_{p^{r+1}} \rightarrow M_{p}$, a diagram (23) can exist for $r+1$ only if it exists for $r$. Suppose then that we have the diagram (23) and thus $\chi$ represents a class in $E_{r}^{*}\left(X ; Z_{p}\right)$. By (8)(d) we get an 
expanded commutative diagram

$$
\begin{array}{rrrr}
E \wedge M_{p^{\prime}} & \stackrel{1 \wedge \pi_{p^{r}}}{\rightarrow} & E \wedge S^{1} \\
x \neg & \downarrow 1 \wedge \overline{p^{r-1}} & \\
X \stackrel{x}{\rightarrow} E \wedge M_{p} \stackrel{1 \wedge \pi_{p}}{\rightarrow-1} & E \wedge S^{1}
\end{array}
$$

Now $\chi$ represents a class in $E_{r+1}^{*}\left(X ; Z_{p}\right)$ if and only if $\left(1 \wedge \pi_{p^{r}}\right) \chi$ is a multiple of $p^{r}$. By (24) this is so if and only if $\left(1 \wedge i_{p} \pi_{p^{\prime}}\right) x_{r}$ is zero. But by Lemma 60 this is so if and only if $\chi_{r}=(1 \wedge \vec{p}) \chi_{r+1}: X \rightarrow E \wedge M_{p^{\prime+1}} \rightarrow E \wedge M_{p^{\prime}}$ for some $\chi_{r+1}: X \rightarrow E \wedge M_{p^{r+1}}$. By (9)(a) this is equivalent to $\chi=\left(1 \wedge \overline{p^{7}} \chi_{r+1}\right.$ : $X \rightarrow E \wedge M_{p^{r+1}} \rightarrow E \wedge M_{p}$, and thus to the existence of a diagram (23) for $r+1$.

LEMMA 62. If $p=2$ then $E_{r}^{*}$ is a $Z_{2}$ module for $r>2$.

Proof. It suffices to prove this for $r=2$. By Lemma 61 it will suffice to show that $2 \cdot \overline{2}: M_{4} \rightarrow M_{2}$ is trivial. This is so because, using an exact sequence argument based on (1), it may be shown that $\left[M_{4}, M_{2}\right]=Z_{2} \oplus Z_{2}$. (Compare with Proposition 9 and see also [3, pp. 81-82].)

Let $\alpha=\alpha_{q}: M \wedge M \rightarrow N$ be chosen to satisfy Propositions 19(a) and 20. We use the various notions of $\S I I .4$.

LEMMA 63. Let $s>r \geqslant 1$ be integers. Then

(a) $k_{1} \alpha_{p^{\prime}}\left(p^{\overline{s-r}} \wedge p^{\overline{s-\eta}}\right)=p^{s-r} k_{1} \alpha_{p s}: M_{p^{s}} \rightarrow S^{1}$,

(b) $\left(p^{s-r} \wedge p^{s-\eta}\left(\alpha_{p s}\right)^{-1} j_{r}=p^{s-r}\left(\alpha_{p r}\right)^{-1} j_{1}: S^{1} \rightarrow M_{p}, \wedge M_{p}\right.$.

Proof. Compare [3, pp. 104-105]. In view of (9) it suffices to consider the case $s=r+1$. For (a), by (8)(a), (18), (17)(a) and (8)(d) we have

$$
\begin{aligned}
k_{1} \alpha_{p}(\bar{p} \wedge \bar{p})\left(1 \wedge i_{p^{\prime+1}}\right) & =k_{1} \alpha_{p^{\prime}}\left(1 \wedge i_{p^{\prime}}\right)(\bar{p} \wedge 1) \\
= & k_{1} j_{0} \bar{p}=\pi_{p} \bar{p}=p \pi_{p^{\prime+1}}=p k_{1} j_{0}=p k_{1} \alpha_{p^{\prime+1}}\left(1 \wedge i_{p^{r+1}}\right) .
\end{aligned}
$$

By (1) we see then that

$$
k_{1} \alpha_{p r}(\bar{p} \wedge \bar{p})=p k_{1} \alpha_{p^{r+1}}+\lambda\left(1 \wedge \pi_{p^{r+1}}\right)
$$

for some $\lambda \in\left[S M_{p^{r+1}}, S^{1}\right]$. By (10), $\lambda=0$ if $p$ is odd, so we are done in this case. For the $p=2$ case, see [8, pp. 71].

The proof of (b) is similar and dual.

LEMMA 64. There are commutative diagrams

$$
\begin{array}{llrl}
E \wedge M_{p^{\prime}} & \stackrel{\tau_{p^{\prime}}}{\rightarrow} & M_{p^{\prime}} \wedge M_{p^{\prime}} \\
\downarrow \overline{p^{s-r}} \wedge \overline{p^{s-r}} & & \downarrow 1 \wedge \overline{p^{s-r}} \\
E \wedge M_{p^{\prime}} & \stackrel{\tau_{p^{\prime}}}{\rightarrow} & M_{p^{\prime}} & \wedge M_{p^{\prime}}
\end{array}
$$


Here $s>r \geqslant 1$ are integers, with the case of $p=2$ and $r=1$ allowed only if $[2]_{E}=0$; and $\tau_{p}$ and $\tau_{p}$, are specific multiplication generators determined by Proposition 22.

Proof. Again by (9) it suffices to consider the case $s=r+1$. As previously noted, if $p$ is odd, Proposition 17 determines a specific unique choice of $\tau_{p^{\prime}}$. For $p=2$ and $r \geqslant 2$ there are two choices for $\tau_{p^{\prime}}$ depending on the choice of $\alpha_{p}$, while for $p=2$ and $r=1$ there may be many choices for $\tau_{p^{\prime}}$.

We proceed inductively on $r$, the inductive procedure being necessary only to cover the case of $p=2$. We begin when $p=2$ by choosing any $\tau_{2}$ or $\tau_{4}$, along with $\alpha_{2}$ or $\alpha_{4}$ (depending on whether or not $[\eta]_{E}=0$ ), satisfying Proposition 22.

We suppose now that $\tau_{p^{1}}, \ldots, \tau_{p^{\prime}}$ have been chosen so that (25) commutes with $\tau_{p^{k+1}}$ and $\tau_{p^{k}}$ in place of $\tau_{p^{s}}$ and $\tau_{p^{\prime}}$ for $k<r$. We want $\tau_{p^{r+1}}$ so that (25) commutes with $s=r+1$. As previously noted, we have no choice when $p$ is odd. When $p=2$ we have two choices for $\tau_{p^{r+1}}$ (and $\alpha_{p r+1}$ ). Choose one arbitrarily, reserving the right to change the choice later.

From the proof of Proposition 22 we have

$$
\tau_{p^{r+1}}=p_{1} \alpha_{p^{r+1}}: M_{p^{r+1}} \wedge M_{p^{r+1}} \rightarrow M_{p^{r+1}} \vee S M_{p^{r+1}} \rightarrow M_{p^{r+1}}
$$

Thus

$$
(1 \wedge \bar{p}) \tau_{p^{r+1}}=(L \wedge 1) \bar{p} p_{1} \alpha_{p^{r+1}}: M_{p^{r+1}} \wedge M_{p^{r+1}} \rightarrow M_{p^{r+1}}
$$

On the other hand,

$$
\begin{aligned}
\tau_{p^{\prime}}(\bar{p} \wedge \bar{p})\left(\alpha_{p^{\prime+1}}\right)^{-1} n_{1} & =\tau_{p^{\prime}}(\bar{p} \wedge \bar{p})\left(1 \wedge i_{p^{\prime+1}}\right) \\
& =\tau_{p^{\prime}}\left(1 \wedge i_{p^{\prime}}\right) \bar{p}=(L \wedge 1) \bar{p}: M_{p^{r+1}} \rightarrow E \wedge M_{p^{\prime \prime}}
\end{aligned}
$$

It follows that it suffices to prove that $\tau_{p^{\prime}}(p \wedge p)\left(\alpha_{p^{r+1}}\right)^{-1} n_{2}=0: S M_{p^{r+1}} \rightarrow E$ $\wedge M_{p^{r}}$. For the details of this proof (possibly changing the choice of $\tau_{p^{r+1}}$ if $p=2)$, see [8, pp. 73-74].

THEOREM 65. (a) Suppose $[\eta]_{E}=0$ if $p=2$. For $r>1$ there is a multiplication $m_{p}: E_{r}^{i}\left(X ; Z_{p}\right) \otimes E_{r}^{j}\left(Y ; Z_{p}\right) \rightarrow E_{r}^{i+j}\left(X \wedge Y ; Z_{p}\right)$. ( $m_{p}$ is bilinear, natural, and has a two-sided unit.) $m_{1}$ is a compatible multiplication on $\bmod p$ cohomology. $m_{r+1}$ is induced by $m_{r}$ for all $r$. Thus, in the obvious sense, each $m_{r}$ is compatible with $\mu$.

(b) $d_{r}$ is an antiderivation with respect to $m_{r}$.

(c) The $m_{r}$ 's are commutative for $p$ odd and for $p=2$ and $r \geqslant 2 . m_{1}$ is also commutative for $p=2$ if $[\bar{\eta}]_{E}=0$.

(d) The $m_{r}$ 's are associative if $p \neq 3$ or if $\left[i_{3} \nu\right]=0$.

Proof. We choose multiplication generators $\tau_{p}$, satisfying Lemma 64. Let $\mu_{p}$, be the corresponding multiplications. Let $m_{1}=\mu_{p}$. We shall show induc- 
tively that $m_{1}$ induces successive multiplications $m_{r}$ on $E_{r}$ for $r \geqslant 2$.

Suppose we have $m_{r}$. Suppose we have cohomology classes represented by $\chi: X \rightarrow E \wedge M_{p}$ and $\chi^{\prime}: Y \rightarrow E \wedge M_{p}$ (of arbitrary degrees) which represent cocycles of $E_{r}$, and thus elements of $E_{r+1}$. Choose $\chi_{r+1}: X \rightarrow E \wedge M_{p^{r+1}}$ and $\chi_{r+1}^{\prime}: Y \rightarrow E \wedge M_{p^{r+1}}$ as given by Lemma 61. Then, by Lemma 64,

$$
\mu_{p}\left(\chi \wedge \chi^{\prime}\right)=\left(1 \wedge \bar{p}^{\prime}\right) \mu_{p^{r+1}}\left(\chi \wedge \chi_{r}^{\prime}\right)
$$

Thus $\mu_{p}\left(\chi \wedge \chi^{\prime}\right)$ represents a cocycle in $E_{r}$. Thus $m_{r+1}$, which we are in the process of defining (we still must show that the product of a cocycle and a coboundary in $E_{r}$ is a coboundary), may by Lemmas 61 and 64 be regarded as induced by either $\mu_{p}$ or $\mu_{p \text { r. }}$.

Now suppose $\chi: X \rightarrow E \wedge M_{p}$ represents a cocycle in $E_{r}$ and $\chi^{\prime}: Y \rightarrow E \wedge$ $M_{p}$ represents any class $X^{\prime}$ in $E_{r}$. Choose $\chi_{r+1}$ as above, and choose $\chi_{r}^{\prime}$ : $Y \rightarrow E \wedge M_{p^{r}}$ as in Lemma 61. Let $\chi_{r}(1 \wedge \vec{p}) \chi_{r+1}: X \rightarrow E \wedge M_{p^{r}}$. Consider the following diagram, which by $(8)$ is commutative.

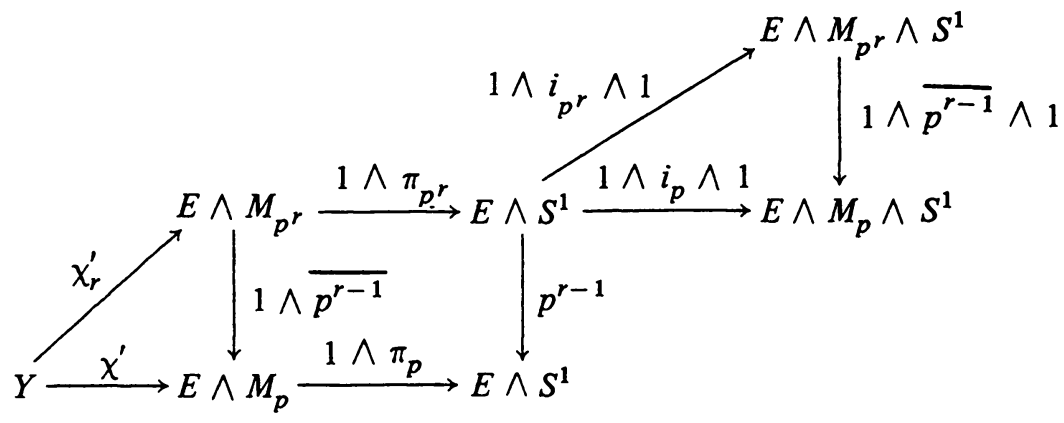

From the diagram we see that $\left(1 \wedge i_{p} \wedge 1\right)\left(1 \wedge \pi_{p^{\prime}}\right) \chi_{r}^{\prime}=\left(1 \wedge \overline{p^{r-1}} \wedge\right.$ 1) $\beta_{p} \cdot \chi_{r}^{\prime}$ represents $d_{r}\left(\chi^{\prime}\right)$, and more generally, that coboundaries in $E_{r}$ are represented by morphism $\left(1 \wedge \overline{p^{r-1}}\right) \beta_{p}, \lambda, \lambda: Y \rightarrow E \wedge M_{p r}$. Since $\mu_{p}$, is Bockstein, we have

$$
\begin{aligned}
& \left(1 \wedge \overline{p^{r-1}}\right) \mu_{p^{\prime}}\left(\chi_{r} \wedge \beta_{p^{\prime}} \chi_{r}^{\prime}\right) \\
& \quad=\left(1 \wedge \overline{p^{r-1}}\right) \beta_{p^{\prime} \mu_{p^{\prime}}}\left(\chi_{r} \wedge \chi_{r}^{\prime}\right) \pm\left(1 \wedge \overline{p^{r-1}}\right) \mu_{p^{\prime}}\left(\beta_{p^{\prime}} \chi_{r} \wedge \chi_{r}^{\prime}\right)
\end{aligned}
$$

but

$$
\left(1 \wedge \overline{p^{r-1}}\right) \mu_{p^{r}}\left(\beta_{p^{\prime}} \chi \wedge \chi_{r}^{\prime}\right)=\mu_{p}\left(\left(1 \wedge \overline{p^{r-1}} \wedge 1\right) \beta_{p^{\prime}} \chi \wedge\left(1 \wedge \overline{p^{r-1}}\right) \chi^{\prime}\right)=0
$$

by Lemma 64, the above remarks and the fact that $x$ is a cocycle. Thus also by the remarks above, the right side of (26) represents a coboundary. Thus $m_{r+1}$ is as desired. 
(b) follows from our representation of coboundaries stated above, together with the fact that $\mu_{p}$, is Bockstein.

(c) and (d) follow from the known properties of $\mu_{p}$ and $\mu_{p}$.

\section{REFERENCES}

1. J. F. Adams, On the groups J(X). IV, Topology 5 (1966), 21-71. MR 33 \#6628.

2. Stable homotopy and generalized homology, Univ. Chicago Press, Chicago and London, 1974. MR 53 \#6534.

3. S. Araki and H. Toda, Multiplicative structures in $\bmod q$ cohomology theories. I, Osaka J. Math. 2 (1965), 71-115. MR 32 \#449.

4. __ Multiplicative structures in mod $q$ cohomology theories. II, Osaka J. Math. 3 (1966), 81-120. MR 34 \#2003.

5. P. E. Conner and E. E. Floyd, The relation of cobordism to $K$-theories, Lecture Notes in Math., vol. 28, Springer-Verlag, Berlin and New York, 1966. MR 35 \# 7344.

6. __ Torsion in SU-bordism, Mem. Amer. Math. Soc. no. 60, 1966. MR 32 \#6471.

7. A. Liulevicious, Notes on homotopy of Thom spectra, Amer. J. Math. 80 (1964), 1-16. MR 29 \#4060.

8. A. F. Martin, Multiplications on mod q cohomology theories, Thesis, Yale University, 1977.

9. C. R. F. Maunder, mod $p$ cohomology theories and the Bockstein spectral sequence, Proc. Cambridge Philos. Soc. 63 (1967), 23-43. MR 35 \#2276.

Department of Mathematical Sciences, Nortfiern IllnNoIs UntverstTy, DeKalb, Illinots 60115 